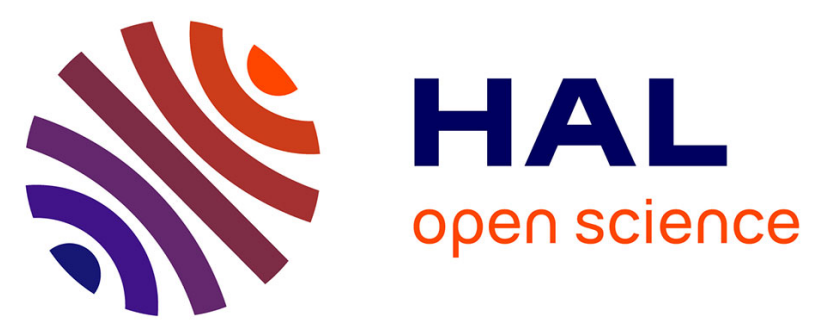

\title{
Finite-temperature and finite-time scaling of the directed polymer free-energy with respect to its geometrical fluctuations
}

Elisabeth Agoritsas, Sebastian Bustingorry, Vivien Lecomte, Gregory Schehr, Thierry Giamarchi

\section{To cite this version:}

Elisabeth Agoritsas, Sebastian Bustingorry, Vivien Lecomte, Gregory Schehr, Thierry Giamarchi. Finite-temperature and finite-time scaling of the directed polymer free-energy with respect to its geometrical fluctuations. Physical Review E : Statistical, Nonlinear, and Soft Matter Physics, 2012, 86 (3), pp.031144. 10.1103/PhysRevE.86.031144 . hal-00713432

\section{HAL Id: hal-00713432 \\ https://hal.science/hal-00713432}

Submitted on 27 Jan 2016

HAL is a multi-disciplinary open access archive for the deposit and dissemination of scientific research documents, whether they are published or not. The documents may come from teaching and research institutions in France or abroad, or from public or private research centers.
L'archive ouverte pluridisciplinaire HAL, est destinée au dépôt et à la diffusion de documents scientifiques de niveau recherche, publiés ou non, émanant des établissements d'enseignement et de recherche français ou étrangers, des laboratoires publics ou privés. 


\title{
Finite-temperature and finite-time scaling of the directed polymer free energy with respect to its geometrical fluctuations
}

\author{
Elisabeth Agoritsas, ${ }^{1}$ Sebastian Bustingorry, ${ }^{2}$ Vivien Lecomte, ${ }^{3}$ Grégory Schehr,${ }^{4}$ and Thierry Giamarchi ${ }^{1}$ \\ ${ }^{1}$ DPMC-MaNEP, Université de Genève, 24 quai Ernest Ansermet, 1211 Genève, Switzerland \\ ${ }^{2}$ CONICET, Centro Atómico Bariloche, 8400 San Carlos de Bariloche, Río Negro, Argentina \\ ${ }^{3}$ Laboratoire de Probabilités et Modèles Aléatoires (CNRS UMR 7599), Universités Paris VI \& Paris VII, site Chevaleret, \\ 175 rue du Chevaleret, 75013 Paris, France \\ ${ }^{4}$ Laboratoire de Physique Théorique et Modèles Statistiques (UMR du CNRS 8626), Université Paris Sud, 91405 Orsay Cedex, France
}

(Received 28 June 2012; published 27 September 2012)

\begin{abstract}
We study the fluctuations of the directed polymer in $1+1$ dimensions in a Gaussian random environment with a finite correlation length $\xi$ and at finite temperature. We address the correspondence between the geometrical transverse fluctuations of the directed polymer, described by its roughness, and the fluctuations of its free energy, characterized by its two-point correlator. Analytical arguments are provided in favor of a generic scaling law between those quantities, at finite time, nonvanishing $\xi$, and explicit temperature dependence. Numerical results are in good agreement both for simulations on the discrete directed polymer and on a continuous directed polymer (with short-range correlated disorder). Applications to recent experiments on liquid crystals are discussed.
\end{abstract}

DOI: 10.1103/PhysRevE.86.031144

PACS number(s): 05.40.-a, 05.20.-y, 68.35.Ct, 46.65.+g

\section{INTRODUCTION}

Brownian particles have provided in physics one of the first example of systems whose geometrical properties differ radically from those encountered in regular classical mechanics: Instead of following "smooth" (differentiable) trajectories, those particles follow continuous but "rough" (nondifferentiable) paths due to the persistent stochastic thermal forces they withstand at finite temperature. This phenomenon is described in statistical mechanics by a random walk, whose geometrical self-similarity at large scale is depicted by scaling laws and scaling exponents. These scalings are known to be distinctive features of universality classes, gathering different physical phenomena sharing a common representation. A natural question regarding such random paths pertains to the influence of the environment: How does a path in a uniform medium differ, for instance, from a path in a medium with random inhomogeneities? And how do such differences manifest in scaling properties?

Beyond particle trajectories, those paths also describe generic interfaces or random manifolds between distinct phases. Example systems include imbibition fronts [1,2], wetting and spreading interfaces $[3,4]$, cracks propagating in paper [5], avalanches of pinned interfaces [6,7], burning fronts $[8,9]$, interfaces in magnetic [10-12] or ferroelectric materials $[13,14]$, and generic growth phenomena $[15,16]$. A wide selection of those systems, although ranging from microscopic to macroscopic scale and presenting a large variety of microphysics, have been described as generic disordered elastic systems (see Refs. [17-23] for reviews).

One class of random walk has received close attention in the past decades, the one-dimensional Kardar-Parisi-Zhang (KPZ) [24] universality class (see Refs. [25,26] for recent reviews), since it is related to questions of extremely varied nature [27] ranging from Burgers equation in hydrodynamics [28], directed polymers in random media $[29,30]$ to the parabolic Anderson model [31], eigenvalues of random matrices [32-34], vicious walkers [35-38], dynamics of cold atoms [39], and transport in one-dimensional stochastic [40] or deterministic models [41].

Here we address the link between the scaling properties of the geometry of the directed polymer (through its scaledependent roughness) and the scaling of its free-energy fluctuations. We examine in particular the role of temperature for those scalings and its possible interplay with finite disorder correlation length, especially at finite time.

More precisely, let us denote by $t$ the direction of time of the growing directed polymer (DP), which also represents the "scale" at which a generic interface is examined, and by $y$ the transverse direction in which fluctuations occur (see Fig. 1). One important aspect of the KPZ universality class is that the fluctuations of the (suitably centered) free energy $\bar{F}(t, y)$ are expected to behave as follows in the large time asymptotics:

$$
\bar{F}(t, y) \stackrel{(t \rightarrow \infty)}{\sim} a t^{\frac{1}{3}} \chi\left(\frac{y}{b t^{\frac{2}{3}}}\right),
$$

where $a$ and $b$ are constants which depend on the physical parameters of the system (e.g., temperature $T$, elasticity $c$, disorder strength $D$, and disorder correlation length $\xi$ in the model we use; see Sec. II), and $\chi(\bar{y})$ is a $t$-independent "random variable" whose distribution characterizes the fluctuations of $y$-dependent observables. Note that in some systems, e.g., liquid crystals, the quantity $\bar{F}(t, y)$ scaling as (1) is, up to a translation, the height of an interface and not its free energy [42-44]. For a directed polymer with fixed endpoints (as depicted in Figs. 1 and 2) and with uncorrelated random environment $(\xi=0)$ it has been shown $[45,46]$ that $\chi(\bar{y})$, considered as a (stochastic) function of $\bar{y}$, is equivalent in distribution to the so-called Airy 2 process (minus a parabola), which is a stationary determinantal point process. It follows from this relation (1) that, in the large $t$ limit, the fluctuations of $\bar{F}(t, y)$, at fixed $y$, are described by the Tracy-Widom distribution $\mathcal{F}_{2}$ [47]. The latter distribution $\mathcal{F}_{2}$, which can be written as a Fredholm determinant involving the Airy kernel, describes the fluctuations of the largest eigenvalue of random matrices belonging to the Gaussian Unitary Ensemble (GUE). 


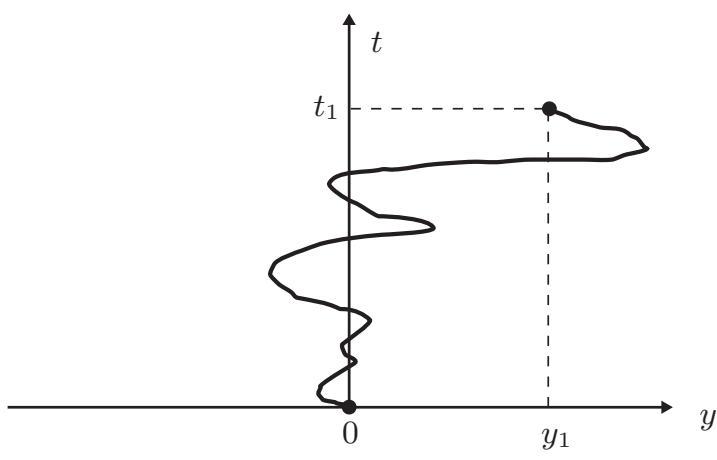

FIG. 1. Schematic view of a continuous directed polymer of trajectory $y(t)$ starting in $y=0$ at time 0 and arriving in $y_{1}$ at time $t_{1}$, in a quenched random potential $V(t, y)$. These point-to-point configurations correspond to the "droplet geometry" of growth models.

Still at $\xi=0$, remarkably, it was shown that at finite time $t$ the distribution of $\bar{F}(t, y)$, for fixed $y$, can still be written as a Fredholm determinant, which involves a nontrivial finite $t$ generalization of the Airy kernel [48-51].

In this paper we focus on the effects of finite temperature and finite disorder correlation length $(\xi>0$ or discrete DP) at finite time for which we propose a generalized scaling relation which reads formally

$$
\bar{F}(t, y) \sim \widetilde{a}[\sqrt{B(t)}]^{\frac{1}{2}} \chi\left(\frac{y}{\widetilde{b} \sqrt{B(t)}}\right),
$$

where $B(t)$ is the roughness of the directed polymer, defined as the second cumulant of the transverse fluctuations along $y$ at fixed time $t$. A more accurate statement is made in Sec. III B in terms of the two-point correlator of $\bar{F}(t, y)$; see Eq. (22). The large time asymptotics (1) is then recovered from $B(t) \stackrel{(t \rightarrow \infty)}{\sim} t^{\frac{4}{3}}$. Our motivation for investigating the case $\xi>0$ comes from the study of physical or chemical experiments where the disordered potential always presents a finite correlation length, which, albeit microscopic and often inaccessible to direct measurement, may still induce observable effects at large scales [23]. In particular, we probe the dependence of the constants $\widetilde{a}, \widetilde{b}$ on the system parameters by distinguishing a high- and a low-temperature regime due to $\xi>0$. The appearance of those regimes generalizes the ones affecting some specific observables (such as the roughness of the interface, studied in Refs. [52,53]).

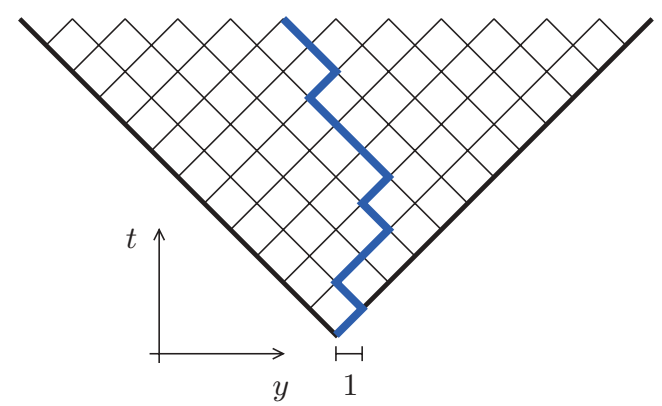

FIG. 2. (Color online) Geometry of the discrete DP model we consider. Allowed steps are $y(t+1)-y(t)= \pm 1$. The longitudinal and transverse axes correspond to the directions $t$ and $y$, respectively.
The directed polymer model we study is defined in Sec. II and the generalized scaling form we propose is presented and discussed analytically in Sec. III. Numerical results are gathered in Sec. IV for the discrete DP and Sec. V for its continuous version. We discuss experimental implications and draw our conclusions in Sec. VI. The details of some computations are gathered in the Appendices.

\section{MODEL AND SCOPE OF THE STUDY}

\section{A. DP formulation}

We focus on the directed polymer formulation: the trajectory of the polymer is described by a continuous coordinate $y(t)$, starting from $y=0$ at $t=0$ (see Fig. 1), and growing in a random potential $V(t, y)$ along the direction $t$. The energy of a trajectory $y(t)$ of duration $t_{1}$ is the sum of elastic and disorder contributions:

$$
\mathcal{H}\left[y, V ; t_{1}\right]=\int_{0}^{t_{1}} d t\left\{\frac{c}{2}\left[\partial_{t} y(t)\right]^{2}+V(t, y(t))\right\} .
$$

The first term flattens the polymer by penalizing its deformations (with an intensity encoded in the elastic constant $c$ ), while the second term tends to deform it. At fixed disorder $V$, the weight of all trajectories starting from 0 and arriving in $y_{1}$ at time $t_{1}$ is given by the path integral

$$
Z_{V}\left(t_{1}, y_{1}\right)=\int_{y(0)=0}^{y\left(t_{1}\right)=y_{1}} \mathcal{D} y(t) e^{-\frac{1}{T} \mathcal{H}\left[y, V ; t_{1}\right]},
$$

(we set the Boltzmann constant equal to 1 and denote thereafter the inverse temperature by $\beta=\frac{1}{T}$ ). The mean value of an observable $O$ depending on the value of $y$ at final time $t$ reads, at fixed disorder

$$
\langle O[y(t)]\rangle_{V}=\frac{\int d y O(y) Z_{V}(t, y)}{\int d y Z_{V}(t, y)} .
$$

Here and hereafter, the integrals $\int d y$ over the DP endpoint run by convention on the real line. We also consider a discrete version of the same system, illustrated in Fig. 2 and described in Sec. IV.

The weight $Z_{V}(t, y)$ is not normalized to unity [ $\int d y Z_{V}(t, y) \neq 1$ in general], but the path integral (4) is chosen so that at zero disorder $\int d y Z_{V \equiv 0}(t, y)=1$ at all times. With this choice of normalization, it is known from the Feynman-Kac formula [54-57] (see also Ref. [58] for a discussion) that the weight $Z_{V}(t, y)$ evolves according to the "stochastic heat equation" [59-61]

$$
\partial_{t} Z_{V}(t, y)=\frac{T}{2 c} \partial_{y}^{2} Z_{V}(t, y)-\frac{1}{T} V(t, y) Z_{V}(t, y),
$$

while the free energy $F_{V}(t, y)=-T \log Z_{V}(t, y)$ obeys the Kardar-Parisi-Zhang equation [24,56]

$$
\partial_{t} F_{V}(t, y)=\frac{T}{2 c} \partial_{y}^{2} F_{V}(t, y)-\frac{1}{2 c}\left[\partial_{y} F_{V}(t, y)\right]^{2}+V(t, y) .
$$

The presence of the so-called nonlinear KPZ term makes the study of this equation particularly difficult; in particular the typical extension of the excursions along the direction $y$ does not scale diffusively at large times (i.e., $y \sim t^{\frac{1}{2}}$ ) but superdiffusively ( $y \sim t^{\zeta}$ where $\zeta=\frac{2}{3}$ is the KPZ exponent) $[24,28,32,45,56,62]$. 
The distribution of the random potential $V(t, y)$ determines the statistical properties of the free energy. We denote by an overline $\overline{\langle O[y(t)]\rangle_{V}}$ the statistical average over the disorder $V$. One case is well understood: When $V(t, y)$ is a centered Gaussian uncorrelated random potential $(\xi=0)$, that is, when $V(t, y)$ has a Gaussian distribution with zero mean and twopoint correlator

$$
\overline{V(t, y) V\left(t^{\prime}, y^{\prime}\right)}=D \delta\left(t^{\prime}-t\right) \delta\left(y^{\prime}-y\right),
$$

it is known for long [56] that the infinite-time distribution of the free energy is that of a two-sided Brownian walk:

$$
\operatorname{Prob}[F(y)] \propto \exp \left\{-\frac{1}{2} \frac{T}{c D} \int d y\left[\partial_{y} F(y)\right]^{2}\right\} .
$$

In other words, $F_{V}(t, y)$ has a steady-state Gaussian distribution whose correlator reads:

$$
\overline{\left[F_{V}\left(t, y^{\prime}\right)-F_{V}(t, y)\right]^{2}} \underset{t \rightarrow \infty}{\longrightarrow} \frac{c D}{T}\left|y^{\prime}-y\right| .
$$

The value $\frac{2}{3}$ for the KPZ exponent is known since the work of Henley, Huse, and Fisher [56] and Kardar, Parisi, and Zhang [24] and Kardar [62,63], but this result has been proven in a mathematical framework much later $[32,45]$. The free-energy distribution has recently been determined by a variety of methods both in the physics $[48,51,64]$ and in the mathematics [49,50,65] communities (see Ref. [66] for a review).

\section{B. Generalization of the free-energy fluctuation scaling}

In the large- but finite-time limit, Prähofer and Spohn [45] have shown that at $\xi=0$ the correlator

$$
C(t, y)=\overline{\left[F_{V}(t, y)-F_{V}(t, 0)\right]^{2}}
$$

obeys the following scaling relation:

$$
C(t, y)=t^{\frac{2}{3}} \tilde{g}_{\mathrm{PS}}\left(y t^{-\frac{2}{3}}\right),
$$

with $\tilde{g}_{\mathrm{PS}}(\bar{y}) \sim \frac{c D}{T}|\bar{y}|$ for small $|\bar{y}|$ [which thus gives (10) in the limit $t \rightarrow \infty$ ] while $\tilde{g}_{\mathrm{PS}}(\bar{y})$ saturates to a constant at large $|\bar{y}|$. This function $\tilde{g}_{\mathrm{PS}}(\bar{y})$ is, up to nonuniversal longitudinal and transverse length scales, the mean square displacement of the Airy $_{2}$ process discussed below in Eq. (42).

Our aim in this paper is to propose and test an extension of the scaling relation (12) (i) at finite time and/or (ii) for a disorder $V(t, y)$ presenting short-range correlations. Much less is known in those two cases. To implement shortrange correlations (on a transverse scale $\xi$ ) in the disorder distribution, we assume that it is still zero-mean Gaussian distributed with correlations of the form

$$
\overline{V(t, y) V\left(t^{\prime}, y^{\prime}\right)}=D \delta\left(t^{\prime}-t\right) R_{\xi}\left(y^{\prime}-y\right) .
$$

Such correlations in direction $y$ on a range of order $\xi$ are described by a rounded delta peak $R_{\xi}(y)$, normalized to unity [ $\left.\int d y R_{\xi}(y)=1\right]$ and by the strength $D$ of the disorder. Note that even for $\xi>0$ the distribution of $V$ is invariant by translation along $y$, as in the uncorrelated case (8) (which corresponds to $\xi=0$ ). For simplicity we may assume in explicit examples that $R_{\xi}(y)$ is a normalized Gaussian of variance $2 \xi^{2}$ :

$$
R_{\xi}^{\text {Gauss }}(y)=\frac{1}{\sqrt{4 \pi \xi^{2}}} e^{-\frac{y^{2}}{4 \xi^{2}}} .
$$

Note that the knowledge of the fluctuations of the free energy $F_{V}$ is not sufficient to retrieve the mean value of physical observables from (5): In general the full distribution of $F_{V}$ is required. The free-energy fluctuations described by the correlator $C(t, y)$ still provide physically relevant information, for instance, combined to scaling arguments [67], or as the starting point of the "toy-model" approach where the freeenergy distribution is approximated to be Gaussian [53,68-71] (see also Sec. III C).

\section{SCALING OF THE FREE-ENERGY CORRELATOR $\bar{C}_{\xi}(t, y)$}

\section{A. A generalized scaling relation}

Let us first identify a definition of the free-energy correlator which is suitable to study its finite-time scaling. The correlator $\overline{\left[F_{V}\left(t, y_{2}\right)-F_{V}\left(t, y_{1}\right)\right]^{2}}$ is invariant by translation along $y$ only in the infinite-time limit (10). To extend this property at finite time, one may take advantage of the "statistical tilt symmetry" (STS) [72-74] of the model which ensures that the free-energy splits into two contributions (see, e.g., Ref. [58] for a derivation at nonzero $\xi$ ):

$$
F_{V}(t, y)=\underbrace{c \frac{y^{2}}{2 t}+\frac{T}{2} \log \frac{2 \pi T t}{c}}_{\text {elastic contribution }}+\underbrace{\bar{F}_{V}(t, y)}_{\begin{array}{c}
\text { disorder } \\
\text { contribution }
\end{array}}
$$

where $c \frac{y^{2}}{2 t}+\frac{T}{2} \log \frac{2 \pi T t}{c}$ is the elastic contribution, which fully captures the initial condition, while $\bar{F}_{V}(t, y)$ is invariant by translation along $y$ in distribution [in mathematical terms [49] $\bar{F}_{V}(t, y)$ is "stationary" along $y$ ]. It represents the contribution of the disordered potential to the free energy, since $\left.\bar{F}_{V}(t, y)\right|_{V \equiv 0}=0$ by definition. Note also from (15) that the initial condition $Z_{V}(0, y)=\delta(y)$ simply writes $\bar{F}_{V}(0, y)=0$. This form of initial condition is technically different from the "sharp wedge" often considered to pin the polymer in $y=0$ at initial time [50] and which also ensures $Z_{V}(0, y)=\delta(y)$.

This translational invariance allows us to define our correlator of interest:

$$
\bar{C}_{\xi}\left(t, y^{\prime}-y\right)=\overline{\left[\bar{F}_{V}\left(t, y^{\prime}\right)-\bar{F}_{V}(t, y)\right]^{2}},
$$

where we have made explicit the dependence in $\xi$ arising from the distribution of $V$. The STS ensures that (16) depends in $y$ and $y^{\prime}$ only through the difference $y^{\prime}-y$, while the invariance of the distribution of the disorder $V$ by the reflection $V \mapsto-V$ ensures that the function $\bar{C}_{\xi}(t, y)$ is even with respect to its argument $y$. Note that at $\xi=0$, the steady-state result (10) implies

$$
\bar{C}_{\xi=0}(t, y) \underset{t \rightarrow \infty}{\longrightarrow} \frac{c D}{T}|y| .
$$

The correlator $\bar{C}_{\xi}(t, y)$ is actually the connected correlator of the full free energy $F_{V}(t, y)$ (see Appendix A)

$$
\begin{aligned}
\bar{C}_{\xi}\left(t, y^{\prime}-y\right)= & \overline{\left[F_{V}\left(t, y^{\prime}\right)-F_{V}(t, y)\right]^{2}} \\
& -\left[\overline{F_{V}\left(t, y^{\prime}\right)-F_{V}(t, y)}\right]^{2} .
\end{aligned}
$$




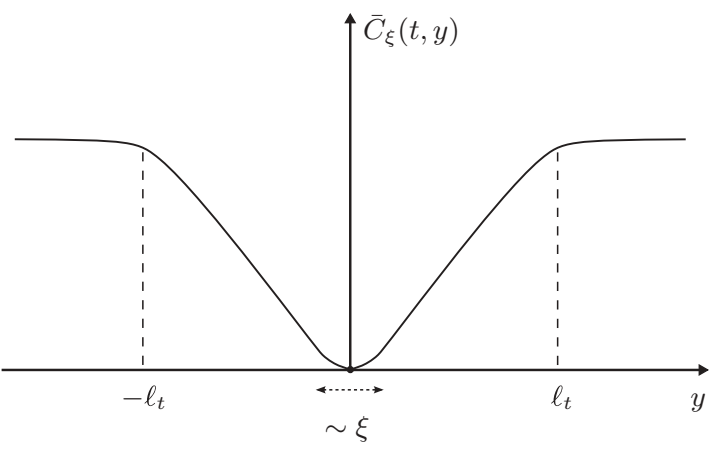

FIG. 3. Schematic plot of the correlator $\bar{C}_{\xi}(t, y)$ of $\bar{F}$, as a function of $y$ at fixed $t$. It is rounded close to the origin $(|y| \lesssim \xi)$, varies as $\widetilde{D}|y|$ for intermediate length $\left(\xi \lesssim|y| \lesssim \ell_{t}\right)$, and goes to a constant at large length $\left(|y| \gtrsim \ell_{t}\right)$.

The expression (16) is useful to explicit the translational invariance, while (18) enables us to consider cases where no decomposition such as (15) is available (as for the discrete DP).

How can we interpret the crossover from the initial condition $\bar{C}_{\xi}(0, y)=0$ to the steady-state result $\bar{C}_{\xi}(\infty, y) \propto|y|$ ? The weight $\propto e^{-\beta c \frac{y^{2}}{2 t}-\beta \bar{F}_{V}(t, y)}$ of a trajectory ending in $y$ at time $t$ depicts a particle of position $y$ (time $t$ being fixed) in an "effective potential" made up of a parabolic potential describing thermal fluctuations and of an "effective disorder" $\bar{F}$, which recapitulates the disorder landscape $V$ perceived by the polymer from its starting point. Heuristically one expects that $\bar{F}$ remains almost flat at initial times since the polymer endpoint $y(t)$ has not explored much of its random environment, while at larger times $\bar{F}$ becomes a "random force potential" with cuspy correlator $\bar{C}_{\xi}(t, y) \propto|y|$, at least on a transverse region $|y| \lesssim \ell_{t}$ of typical size $\ell_{t}$ in which the polymer endpoint has mainly been confined.

We show in Appendix B that this intuitive picture is indeed correct: At all finite times, $\lim _{|y| \rightarrow \infty} \partial_{y} \bar{C}_{\xi}(t, y)=0$. The correlator $\bar{C}_{\xi}(t, y)$ thus has to switch from the absolute value behavior $|y|$ for $|y| \lesssim \ell_{t}$ to a plateau for $|y| \gtrsim \ell_{t}$ (see Fig. 3), at some scale $\ell_{t}$ increasing and diverging with $t$. We assume that the effect of the correlation length $\xi$ is to round $\bar{C}_{\xi}(t, y)$ at small $|y| \lesssim \xi$. The following scaling relation is thus expected to hold:

$$
\bar{C}_{\xi}(t, y)=\ell_{t} \hat{C}_{\ell_{t}^{-1} \xi}\left(\ell_{t}^{-1} y\right),
$$

where the scaling function $\hat{C}_{\xi}(y)$ depends on the physical parameters $c, D, T$, and $\xi$. At zero $\xi$, it is compatible with the large time behavior (12), provided that $\ell_{t} \sim t^{\frac{2}{3}}$ for $t \rightarrow \infty$. We now have to identify the crossover length $\ell_{t}$ at nonzero $\xi$ and finite time.

\section{B. Asymptotic transverse fluctuations}

The variance of the DP endpoint $y(t)$, called the roughness, is the simplest length quantifying the spatial extension of the polymer at a given time $t$ :

$$
B(t)=\overline{\left\langle y(t)^{2}\right\rangle_{V}}=\overline{\frac{\int d y y^{2} Z_{V}(t, y)}{\int d y Z_{V}(t, y)} .}
$$

It is known that the roughness presents at small times a diffusive regime $\left(\zeta_{\text {th }}=\frac{1}{2}\right)$ and at large times a random manifold $(\mathrm{RM})$ superdiffusive regime $\left(\zeta_{\mathrm{RM}}=\frac{2}{3}\right)[24,32,56]$. In terms of power laws of the time $t$, we have the two asymptotic regimes

$$
B(t) \sim \begin{cases}t & \text { for } t \rightarrow 0, \\ t^{\frac{4}{3}} & \text { for } t \rightarrow \infty\end{cases}
$$

(see also Refs. [53,58] when the disorder correlation length $\xi$ is nonzero). We propose that $\ell_{t} \sim \sqrt{B(t)}$ in Eq. (19), in other words,

$$
\bar{C}_{\xi}(t, y)=\sqrt{B(t)} \hat{C}_{\frac{\xi}{\sqrt{B(t)}}}\left(\frac{y}{\sqrt{B(t)}}\right) .
$$

Before testing numerically this scaling law in different models (see Secs. IV and V), we discuss analytical arguments in favor of (22). On one hand, linearizing the dynamics at short time in Eq. (7) one finds that the fluctuations are diffusive: $B(t)=\frac{T t}{c}$. In this approximation, the correlations of the free-energy rescale as follows (see Appendix C):

$$
\bar{C}_{\xi}^{\operatorname{lin}}(t, y)=\frac{c D}{T} \sqrt{B_{\mathrm{th}}(t)} \hat{C}_{\frac{\xi}{\sqrt{B_{\mathrm{th}}(t)}}}^{\operatorname{lin}}\left(\frac{y}{\sqrt{B_{\mathrm{th}}(t)}}\right),
$$

where $B_{\mathrm{th}}(t)=\frac{T t}{c}$ is the thermal roughness. The scaling function $\hat{C}_{\bar{\xi}}^{\operatorname{lin}}(\bar{y})$, given in Eq. (C27), and depending on the properly adimensional variables $\bar{y}$ and $\bar{\xi}$, is independent of the parameters $c, D$, and $T$. The behavior of $\bar{C}_{\xi}^{\operatorname{lin}}(t, y)$ is as qualitatively expected with a rounding on a scale $\xi$, a plateau at large $y$, and a developing linear behavior in between, as plotted in Fig. 4 .

On the other hand, at zero $\xi$ and large time, the result (12) of Prähofer and Spohn is compatible with (22): This corresponds to the RM asymptotics $B(t) \sim t^{\frac{4}{3}}$. To address the RM regime at nonzero $\xi$, we study in the next paragraph a toy-model approach of the DP.

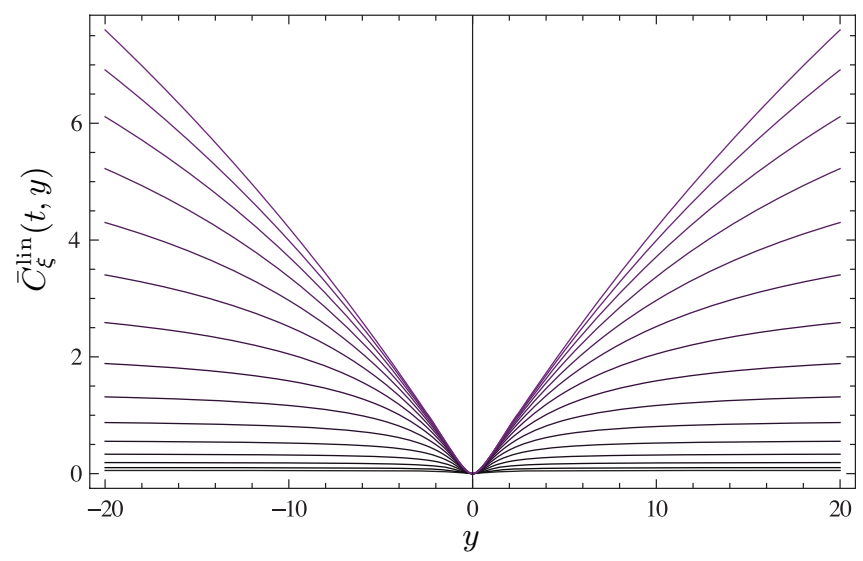

FIG. 4. (Color online) Graph of the correlator $\bar{C}_{\xi}^{\operatorname{lin}}(t, y)$ of $\bar{F}$ obtained in the linear approximation (C23), as a function of $y$ for different times $t$ (parameters are $c=1, D=1, T=1$, and $\xi=0.3$ ). Time increases geometrically from bottom $\left(t=2^{-4}\right)$ to top $\left(t=2^{10}\right)$ curves. 


\section{A winged DP toy model}

In the infinite-time limit and at zero $\xi$, the free energy $F_{V}(t, y)$ is translationally invariant in distribution and has a Gaussian distribution (9). The statistical tilt symmetry expresses that the free energy splits at finite time in two contributions (15), of which only $\bar{F}_{V}(t, y)$ is translationally invariant. The idea of the toy model $[68-70,74]$ is to assume that for large $t$ and finite $\xi$ the distribution of the reduced free energy [denoted $\bar{F}^{\text {toy }}(t, y)$ ] remains in a good approximation Gaussian, with zero mean and correlations

$$
\bar{C}_{\xi}^{\text {toy }}\left(t, y^{\prime}-y\right)=\overline{\left[\bar{F}^{\text {toy }}\left(t, y^{\prime}\right)-\bar{F}^{\text {toy }}(t, y)\right]^{2}} .
$$

It has been shown [53] that the roughness $B(t)$ can be computed in a Gaussian Variational Method (GVM) approximation, for a correlator $\bar{C}_{\xi}^{\text {toy }}(t, y)$ of the Fourier form

$$
\bar{C}_{\xi}^{\text {toy }}(t, y)=\int \frac{d \lambda}{2 \pi} \frac{2}{\lambda^{2}}[1-\cos (\lambda y)] R_{\widetilde{\xi}}^{\mathrm{toy}}(t, \lambda),
$$

with $R_{\widetilde{\xi}}^{\text {toy }}(t, \lambda)=\widetilde{D} e^{-\lambda^{2} \widetilde{\xi}^{2}}$. For $\widetilde{\xi}=0$, this writing yields the $\xi=0$ infinite-time result $(10) \quad \bar{C}(\infty, y)=\widetilde{D}|y|$, provided that $\widetilde{D}=\frac{c D}{T}$. For $\xi>0, R_{\widetilde{\xi}}^{\text {toy }}(t, \lambda)$ encodes for $\bar{F}$ the finite correlation length of the disorder $V$ : The absolute value becomes rounded around the origin up to a scale $\sim \widetilde{\xi}$. The problem of the form (25) of the correlator is that it implies $\bar{C}_{\xi}^{\text {toy }}(t, y) \sim \widetilde{D}|y|$ at large $|y|$, while we have seen that $\bar{C}_{\xi}(t, y)$ goes to a constant in the limit $|y| \rightarrow \infty$ at all finite times $t$.

To overcome the discrepancy between this exact result and the model $R_{\widetilde{\xi}}^{\text {toy }}(t, \lambda)=\widetilde{D} e^{-\lambda^{2} \widetilde{\xi}^{2}}$, one may rather study a correlator $\bar{C}_{\xi}^{\text {toy }}(t, y)$ of the form (25) with additional saturation "wings"

$$
R_{\widetilde{\xi}}^{\text {toy }}(t, \lambda)=\widetilde{D} \frac{\lambda^{2}}{\lambda^{2}+\ell_{t}^{-2}} e^{-\lambda^{2} \widetilde{\xi}^{2}},
$$

which still presents a behavior $\bar{C}^{\text {toy }}(t, y) \sim \widetilde{D}|y|$ at intermediate $y\left(\xi \ll|y| \ll \ell_{t}\right)$, but goes to a constant at large $|y|$ $\left(|y| \gg \ell_{t}\right)$. Note that more generically one can consider a toy correlator of the form

$$
R_{\widetilde{\xi}}^{\text {toy }}(t, \lambda)=\widetilde{D} f_{1}\left(\lambda \ell_{t}\right) f_{2}(\lambda \widetilde{\xi})
$$

in which the rounding due to the finite disorder correlation length appears in the factor $f_{2}(\lambda \widetilde{\xi})$ and the crossover at scale $\ell_{t}$ in the function $f_{1}\left(\lambda \ell_{t}\right)$. ${ }^{1}$ In particular, scaling properties of the roughness arise from the form (27). Note that this form makes sense in principle only when the scales $\ell_{t}$ and $\widetilde{\xi}$ are separated enough (with $\ell_{t}>\widetilde{\xi}$ ). We compute in Appendix D the roughness of this model in the Gaussian Variational Method (GVM) approximation, in the large $\ell_{t}$ limit. The computation yields, in the random manifold regime

$$
B(t)=\left.B(t)\right|_{\ell_{t}^{-1}=0}-\frac{\widetilde{D}}{c^{2} \ell_{t}} t^{2}+O\left(\ell_{t}^{-2}\right),
$$

\footnotetext{
${ }^{1}$ Taking in Eq. (27) $f_{1}(k) \sim k^{2}$ as $k \rightarrow 0$ ensures that $\bar{C}_{\xi}^{\text {toy }}(t, y)$ goes to a constant plateau for $|y| \gg \ell_{t}$ while $f_{1}(k) \sim 1$ as $k \rightarrow \infty$ ensures $\bar{C}_{\xi}^{\text {toy }}(t, y) \sim \tilde{D}|y|$ for $\xi \lesssim|y| \lesssim \ell_{t}$.
}

where the RM roughness in the absence of wings $\left(\ell_{t}^{-1}=0\right)$ is given by

$$
\left.B(t)\right|_{\ell_{t}^{-1}=0}=\frac{3}{2}\left(\frac{2 \widetilde{D}^{2}}{\pi c^{4}}\right)^{\frac{1}{3}} t^{\frac{4}{3}}-\widetilde{\xi}^{2} .
$$

For the scale $\ell_{t}$ not to destroy the $\frac{2}{3}$ exponent of the RM regime, one must have $\ell_{t}$ growing at least as

$$
\ell_{t} \sim\left(\frac{\widetilde{D}}{c^{2}}\right)^{\frac{1}{3}} t^{\frac{2}{3}}
$$

In other words, the wings of $\bar{C}(t, y)$ have to appear at a scale larger than $\sqrt{B(t)}$ in the RM regime.

\section{NUMERICAL SIMULATIONS AT HIGH TEMPERATURE: THE DISCRETE DP}

\section{A. Model}

We use numerical simulations of the DP model in order to test the scaling properties of free-energy fluctuations at finite temperature. Following the geometry described in Fig. 2 we perform numerical simulations of a discrete DP model [63] with the solid-on-solid (SOS) restriction $|y(t+1)-y(t)|=1$. A site-dependent zero-mean uncorrelated Gaussian disorder potential $V_{t, y}$ of intensity $D$ is used:

$$
\overline{V_{t, y} V_{t^{\prime}, y^{\prime}}}=D \delta_{t, t^{\prime}} \delta_{y, y^{\prime}} .
$$

The energy of a given configuration of the DP is given by the sum of the site energies along the path $y(t)$.

Given a disorder realization characterized by $V_{t, y}$, the weight $Z_{t, y}$ of a polymer starting in $(0,0)$ and ending in $(t, y)$ is given by the following recursion:

$$
Z_{t, y}=e^{-\beta V_{t, y}}\left(Z_{t-1, y-1}+Z_{t-1, y+1}\right)
$$

with zero-time initial condition $Z_{0, y}=\delta_{0, y}$. Therefore, the probability to observe a polymer ending in $(t, y)$ is $Z_{t, y} / \sum_{y^{\prime}} Z_{t, y^{\prime}}$. Due to the recursion relation, $Z_{t, y}$ grows exponentially with time $t$. To avoid numerical instability, all weights $Z_{t, y}$ at fixed $t$ are divided by the largest one, which does not change the polymer ending probability. In terms of the weight $Z_{t, y}$ the free energy of the polymer starting in $(0,0)$ and ending in $(t, y)$ is therefore defined as

$$
F_{t, y}=-T \ln \left(\frac{Z_{t, y}}{\sum_{y^{\prime}} Z_{t, y^{\prime}}}\right),
$$

and the free-energy fluctuations are measured in terms of the connected correlation

$$
\bar{C}\left(t, y-y^{\prime}\right)=\overline{\left[F_{t, y}-F_{t, y^{\prime}}\right]^{2}}-\overline{\left[F_{t, y}-F_{t, y^{\prime}}\right]^{2}} .
$$

As discussed in Sec. III A, this definition is equivalent in the continuum to that involving $\bar{F}(t, y)$ in Eq. (16). The index $\xi$ is dropped from $\bar{C}(t, y)$ since the disorder is uncorrelated as in Eq. (8), and since no length scale below the lattice spacing can be considered in this discrete DP model. The roughness is defined as the mean square displacement of the free end, as in 


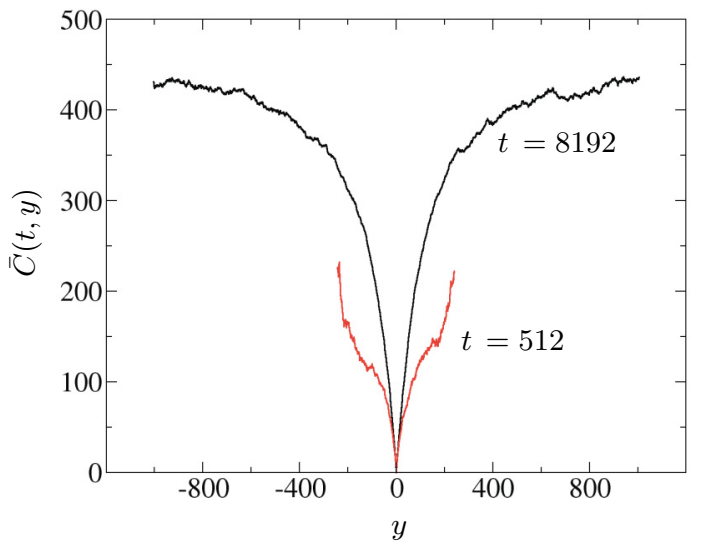

FIG. 5. (Color online) Bare free-energy fluctuations $\bar{C}(t, y)$ for the discrete DP at finite temperature $T=4$ and for two different times $t$. The late increase of $\bar{C}(t, y)$ for $t=512$ is a finite-size effect related to the solid-on-solid restriction of the DP model.

Eq. (20) for the continuous DP

$$
B(t)=\overline{\left\langle y(t)^{2}\right\rangle}=\overline{\sum_{y} y^{2} \frac{Z_{t, y}}{\sum_{y^{\prime}} Z_{t, y^{\prime}}} .}
$$

Finally, note the correspondence between the discrete and continuous parameters [see (E8) with lattice spacings $a$ and $b$ taken to 1]: the continuum model (6) which is the limit of the discrete model (32) in the large size limit has the parameters

temperature $T$, elasticity $c=T, \quad$ disorder $D$,

thus providing a comparison of the temperature dependence of the two models through the correspondence $c=T$. A generic study of the continuum limit of the discrete DP is presented in Appendix E.

\section{B. Results}

In what follows, results are presented for the discrete DP model with $D=1$ and disorder averages are performed on $10^{4}$ disorder realizations. Figure 5 displays typical curves for the free-energy fluctuations $\bar{C}(t, y)$ at a temperature $T=4$. Two different timescales are presented, $t=512$ and $t=8192$, in order to show how the finite size of the system affects free-energy fluctuations. It is clear that one observes for both timescales the characteristic $|y|$ behavior at small transverse length scales and then a crossover to saturation at larger timescales. In addition, when the polymer length is small an increase of $\bar{C}(t, y)$ is observed for large $y$, as can be observed for the $t=512$ data. This last point is a finite-size effect related to the fact that, in contrast to the continuous case, for the discrete DP model used here the polymer endpoint is constrained at all times to remain in the cone $|y(t)| \leqslant t$; see Fig. 2. This finite-size effect only plays a role when analyzing small DP lengths, and in the following we discard this finite-size regime in order to better compare different curves.

We now test the different scaling properties for $\bar{C}(t, y)$. With respect to time $t$ and in the large time limit [45], $\bar{C}(t, y)$ is expected to scale according to

$$
\bar{C}(t, y) \sim t^{\zeta} \hat{C}\left(\frac{y}{t^{\zeta}}\right)
$$

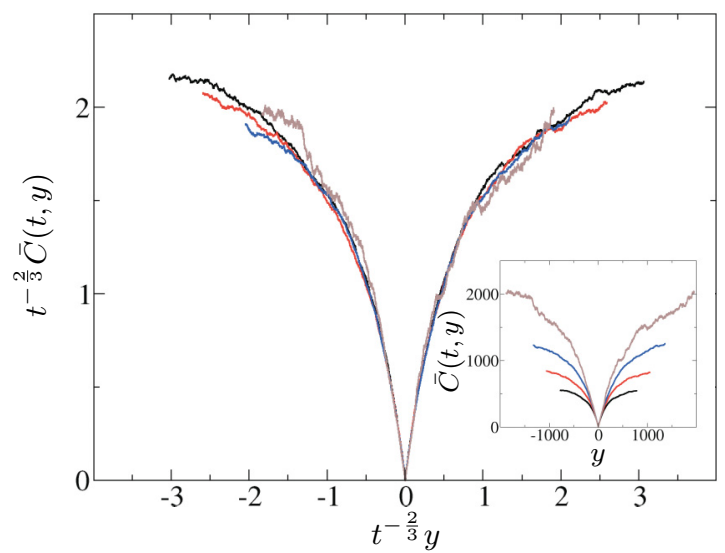

FIG. 6. (Color online) Rescaled free-energy fluctuations $\bar{C}(t, y)$ for the discrete DP at finite low temperature $T=0.5$ and various times, according to the scaling (37) with $\zeta=\zeta_{\mathrm{RM}}=\frac{2}{3}$. The inset shows the bare data $\bar{C}(t, y)$ against $y$ for timescales $t=4096,8192$, 16384,32768 from bottom to top.

with $\zeta=\zeta_{\mathrm{RM}}=\frac{2}{3}$ at large $t$, which corresponds to the scaling relation in Eq. (12). This behavior has been reported in Ref. [69] for a finite low-temperature value $T \approx 0.14(\beta=7)$ and moderated polymer sizes $t=512,1024$. In Fig. 6 we test the scaling given in Eq. (37) for temperature $T=0.5$, which is larger than the one used in Ref. [69] but still in a low-temperature regime (see below). As shown in the figure the scaling works satisfactorily for the large timescales used. This result confirms the scaling probed in Ref. [69] and subsequently analytically obtained in Ref. [45].

In order to go beyond the large time scaling of Eq. (37), we now discuss the temperature dependence of free-energy fluctuations with respect to the roughness. It is first important to properly consider the finite temperature scaling of the roughness, which has been discussed in Refs. [52,53,75]. In the high-temperature regime and within the continuum limit of the DP, the roughness scales asymptotically as

$$
B(t) \sim\left\{\begin{array}{ll}
\frac{T}{c} t^{2 \zeta_{\text {th }}} & \left(t \ll L_{T}\right) \\
\left(\frac{c T}{D}\right)^{2 \mathrm{p}} t^{2 \zeta_{\mathrm{RM}}} & \left(t \gg L_{T}\right)
\end{array} \quad \text { with } \quad L_{T}=\frac{T^{5}}{c D^{2}},\right.
$$

where $\zeta_{\text {th }}=\frac{1}{2}$ is the thermal roughness exponent and $\zeta_{\mathrm{RM}}=\frac{2}{3}$. The temperature dependence of the roughness is described at large scale by the thorn exponent $\mathrm{p}=-\frac{1}{3}$. The thermal length scale $L_{T}$ separates short-scale thermal fluctuations characterized by $\zeta_{\text {th }}$ from large-scale disorder-induced fluctuations of exponent $\zeta_{\mathrm{RM}}$. It has been shown [75] that in the high-temperature regime the thermal length scale grows as $L_{T} \sim T^{1 / \theta_{F}} /\left(c D^{2}\right)$, where $\theta_{F}=\frac{1}{5}$ is the Flory exponent $[52,53]$. In the discrete version of the DP one has $c=T$ [see (36) and Appendix E] and therefore the scaling of the roughness (38) now reads

$$
B(t) \sim\left\{\begin{array}{ll}
t^{2 \zeta_{\text {th }}} & \left(t \ll L_{T}\right) \\
\left(\frac{T^{2}}{D}\right)^{2 \mathrm{p}} t^{2 \zeta_{\mathrm{RM}}} & \left(t \gg L_{T}\right)
\end{array} \quad \text { with } \quad L_{T}=\frac{T^{4}}{D^{2}} .\right.
$$

In this last case, remarkably, the short-time behavior is temperature-independent, while the large time prefactor scales with temperature as $T^{4 \mathrm{p}}=T^{-4 / 3}$. 


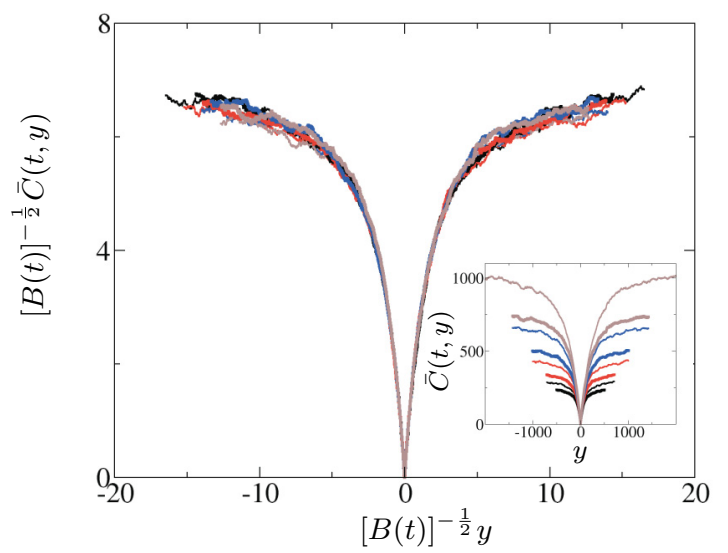

FIG. 7. (Color online) Rescaled free-energy fluctuations $\bar{C}(t, y)$ for the discrete DP at large times and high temperatures, according to the scaling (40). The inset shows the bare data $\bar{C}(t, y)$ against $y$ for timescales $t=4096,8192,16384,32768$ from bottom to top. Thin (thick) lines correspond to $T=4(T=8)$.

These scaling properties of the roughness can be directly incorporated into the scaling properties of free-energy correlations using the whole roughness to define the characteristic transverse scale as in the scaling relation (22), which, in the absence of $\xi$, may be written

$$
\bar{C}(t, y)=\sqrt{B(t)} \hat{C}\left(\frac{y}{\sqrt{B(t)}}\right) .
$$

First note that the inset of Fig. 9 shows how the saturation regime of free-energy fluctuations is reached at smaller transverse length scale $y$ when increasing the temperature. If, as suggested by the scaling relation (40), the crossover is dictated by the time-dependent roughness $B(t)$, this is in agreement with the negative value of the thorn exponent ruling the temperature dependence of the roughness [52,53,75]. The main panel of Fig. 7 illustrates the validity of the scaling relation (40) that incorporates the temperature dependence through the roughness function, in the range of high-temperature roughness.

Since the temperature dependence is included in the roughness $B(t)$, this scaling relation can also be probed at lower temperatures. This is done in Fig. 8, were data corresponding to two low temperatures $T=0.5,1$ and different timescales are collapsed on a single curve. However, the high- and lowtemperature regimes are not necessarily described by the same rescaling function $\hat{C}(\bar{y})$. Figure 9 actually shows, for a single value $t=16384$, that although high- and low-temperature data collapse on a single curve proper to each regime, there is a crossover between these two limiting cases.

As suggested by the scaling relation (23), the slope of the linear initial growth of the free-energy correlator should fully account for the temperature dependence. As extensively discussed in Ref. [58], it can in fact be argued that the $(D, T)$ dependence of the scaling law (22) can be absorbed in a single prefactor $\widetilde{D}$, which in the case of the discrete DP model reads

$$
\bar{C}(t, y)=\widetilde{D} \sqrt{B(t)} \hat{C}^{1}\left(\frac{y}{\sqrt{B(t)}}\right),
$$

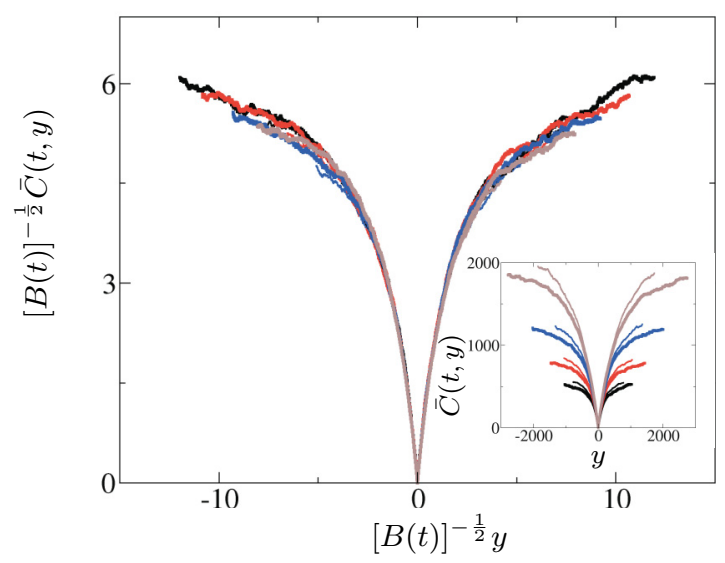

FIG. 8. (Color online) Rescaled free-energy fluctuations $\bar{C}(t, y)$ for the discrete DP at large times and low temperatures, according to the scaling (40). The inset shows the bare data $\bar{C}(t, y)$ against $y$ for timescales $t=4096,8192,16384,32768$ from bottom to top. Thin (thick) lines correspond to $T=0.5(T=1)$.

where $\widetilde{D}=\widetilde{D}(D, T)$ and the function $\hat{C}^{1}(\bar{y})$ is independent of the parameters $D$ and $T$. Indeed, the prefactor $\widetilde{D}$ in Eq. (41) is the slope of the correlator $\bar{C}(t, y)$ in the regime $|y| \lesssim \sqrt{B(t)}$ where $\bar{C}(t, y) \propto|y|$. It is known from the large-time limit at zero $\xi$ that $\widetilde{D}=\frac{c D}{T}$; see (17) [and also (C25) in the diffusive regime]. In the discrete DP case $(c=T), \widetilde{D}$ is thus expected to be temperature independent in the high-temperature regime, $\widetilde{D}=D$, and to behave as $T^{2 / 3}$ in the low-temperature regime [58]. In order to test this, we rescale the data in Fig. 9 onto a single universal master curve as shown in Fig. 10. To this purpose, we fix $\widetilde{D}=D=1$ for $T=4,8$ and ensure the collapse of low-temperature correlators $\bar{C}(t, y)$ by proposing values for the parameter $\widetilde{D} / D$ for $T=0.5,1,2$, which are plotted in the inset of Fig. 10. Although the value of $\widetilde{D}$ is decreasing with $T$ we did not observe the $T^{2 / 3}$ behavior, possibly because we are not reaching the corresponding low-temperature asymptotic regime for the discrete DP model. However, it is remarkable to observe the crossover towards the

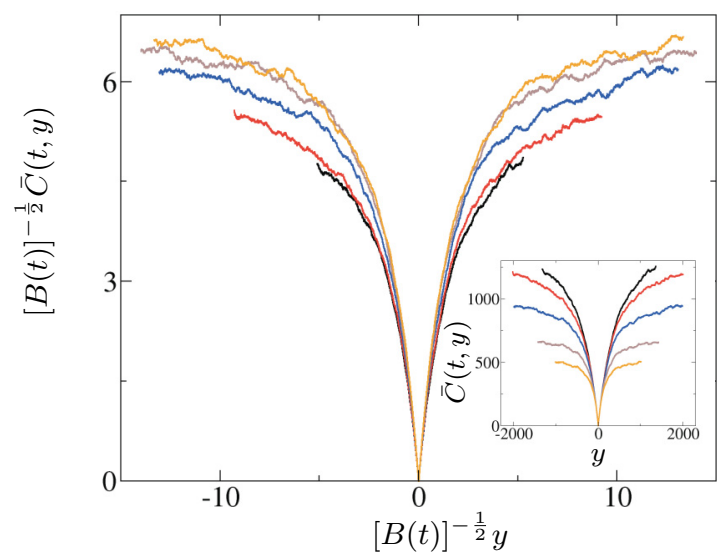

FIG. 9. (Color online) Rescaled free-energy fluctuations $\bar{C}(t, y)$ for the discrete DP at a fixed large time $t=16384$ and from low to high temperatures, according to the scaling (40). The inset shows the bare data $\bar{C}(t, y)$ against $y$. Temperatures are $T=0.5,1,2,4,8$ from top to bottom. 


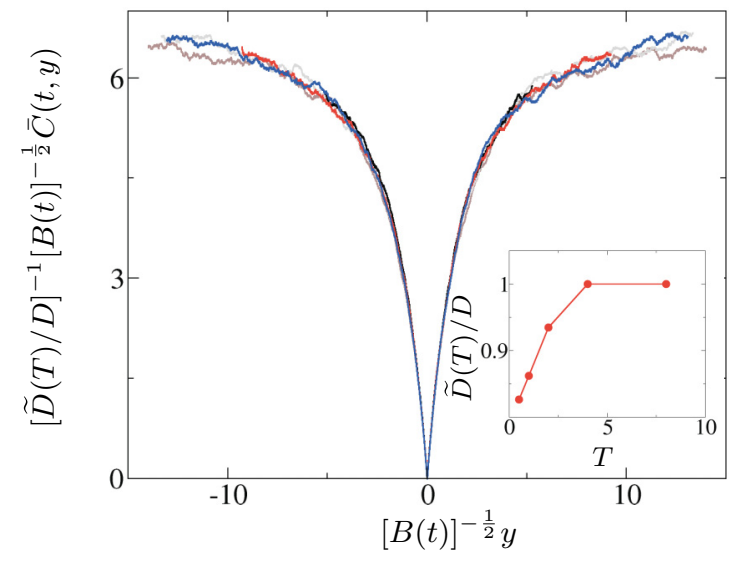

FIG. 10. (Color online) Full temperature-independent rescaled free-energy fluctuations $\bar{C}(t, y)$ for the discrete DP at a fixed large time, according to the scaling (41). Same data as in Fig. 9. The inset shows the parameter $\widetilde{D}$ of (41) as a function of $T$.

low-temperature regime, which reflects the influence of the lattice spacing even though no length scale is defined below it.

In summary, we have shown in this section that numerical simulations of the discrete version of the DP model with the SOS constraint are fully compatible with the scaling arguments suggesting that $\sqrt{B(t)}$ is the relevant transverse scale for the free-energy fluctuations. Moreover, we have also shown that all the temperature dependence can only be accounted for by using, in addition to the proper transverse length scale $\sqrt{B(t)}$ the scale of linear free-energy fluctuations at small $y$, given by the function $\widetilde{D}$. This universal behavior being established, one would naturally expect from the result of Refs. $[45,46]$ in Eq. (12), that the correlator $\hat{C}^{1}(y)$ in Eq. (41) can be expressed in terms of the mean square displacement of the Airy 2 process, $\mathcal{A}_{2}(y)$, namely,

$\hat{C}^{1}(y)=A g_{\mathrm{PS}}(y / B), \quad g_{\mathrm{PS}}(y)=\left\langle\left[\mathcal{A}_{2}(y)-\mathcal{A}_{2}(0)\right]^{2}\right\rangle$,

where $A$ and $B$ are longitudinal and transverse parameterdependent length scales. In Fig. 11 we test this relation (42) by

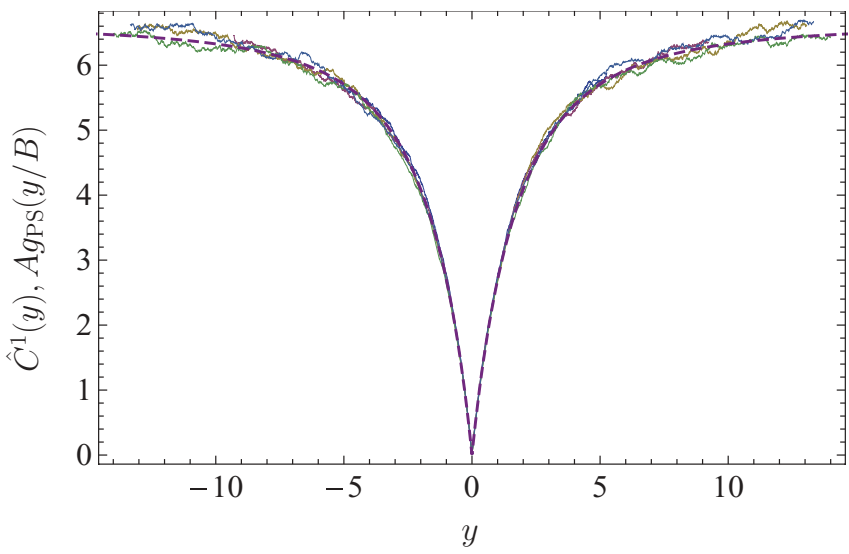

FIG. 11. (Color online) Comparison between $\hat{C}^{1}(y)$ defined in Eq. (41), extracted from the numerical data shown in Fig. 10 and $A g_{\mathrm{PS}}(y / B)$ (dashed line), where $g_{\mathrm{PS}}(y)$ is defined in Eq. (42) while $A$ and $B$ are two fitting parameters (determined to $A \simeq 4.08 \pm 0.05$ and $B \simeq 2.07 \pm 0.06$ ). The numerical evaluation of the exact expression for $g_{\mathrm{PS}}(y)$ was done in Refs. [76,77]. adjusting the parameters $A$ and $B$ using least-square fittings, and we find indeed a very good collapse of our numerical data and the exact expression of $g_{\mathrm{PS}}(y)$ obtained in Ref. [45]. Note that the numerical evaluation of $g_{\mathrm{PS}}(y)$ was done in Refs. [76,77], where precise numerical techniques were developed to compute Fredholm determinants with high precision.

In the following section we extend this analysis to lower temperatures and short-range correlations by studying a continuous version of the DP model.

\section{NUMERICAL SIMULATIONS AT LOWER TEMPERATURE: THE CONTINUOUS DP}

\section{A. Numerical approach}

To probe the implications for the scaling laws of short-range correlations $(\xi>0)$ of the disordered potential $V(t, y)$, we have simulated a continuous version of the directed polymer. Directly integrating the partial differential equation (PDE) (6) for $Z_{V}(t, y)$ is difficult since, especially at low temperature, the weight concentrates exponentially in the most favorable regions for the polymer, preventing the whole space to be embraced. Taking the logarithm and considering the PDE (7) for $F_{V}(t, y)$ is also problematic because of the singular initial condition $Z_{V}(0, y)=\delta(y)$. We took advantage of the STS (15) by directly simulating the PDE for the reduced free energy $\bar{F}_{V}(t, y)$ :

$$
\begin{aligned}
\partial_{t} \bar{F}_{V}(t, y)= & \frac{1}{2 \beta c} \partial_{y}^{2} \bar{F}_{V}(t, y)-\frac{1}{2 c}\left[\partial_{y} \bar{F}_{V}(t, y)\right]^{2} \\
& -\frac{y}{t} \partial_{y} \bar{F}_{V}(t, y)+V(t, y)
\end{aligned}
$$

whose initial condition is simply $\bar{F}_{V}(0, y)=0$. Another benefit of considering $\bar{F}_{V}(t, y)$ instead of $F_{V}(t, y)$ is the removal of the quadratic contribution $c \frac{y^{2}}{2 t}$ which eclipses the disorder contribution in Eq. (6) at short time.

The correlated disordered potential $V(t, y)$ is constructed as follows: Independent random variables $V_{i, j}$ are drawn from a centered Gaussian distribution of variance $D^{\text {grid }}$ on a grid of coordinates $(t, y)=\left(i \xi_{t}, j \xi_{y}\right)$. The continuum $V(t, y)$ is defined as the two-dimensional cubic spline of the $\left\{V_{i, j}\right\}$ on the grid. Its distribution is Gaussian and characterized by its two-point correlator, which can be analytically computed [58] and takes the form

$$
\overline{V(t, y) V\left(t^{\prime}, y^{\prime}\right)}=D R_{\xi_{t}}\left(t^{\prime}-t\right) R_{\xi_{y}}\left(y^{\prime}-y\right),
$$

where $R_{\xi}$ is a normalized smooth delta function of width $\xi$. The amplitude of disorder is $D=D^{\text {grid }} \xi_{t} \xi_{y}$ and our correlation length of interest in the transverse direction is $\xi=\xi_{y}$. In numerical simulations, we took $\xi_{t}=1, \xi_{y}=2, D^{\text {grid }}=4$ (and thus $\xi=2$ and $D=8$ ). Simulations are run on a finite window $\left[-y_{\mathrm{m}}, y_{\mathrm{m}}\right]$ in the transverse direction, outside of which the disorder potential is set to 0 . Besides, since the equation (43) is ill-defined at $t=0$, simulations were run starting at small initial time $t_{0}$ with thermal initial conditions. A complete presentation of the numerical procedure can be found in Ref. [58]. 


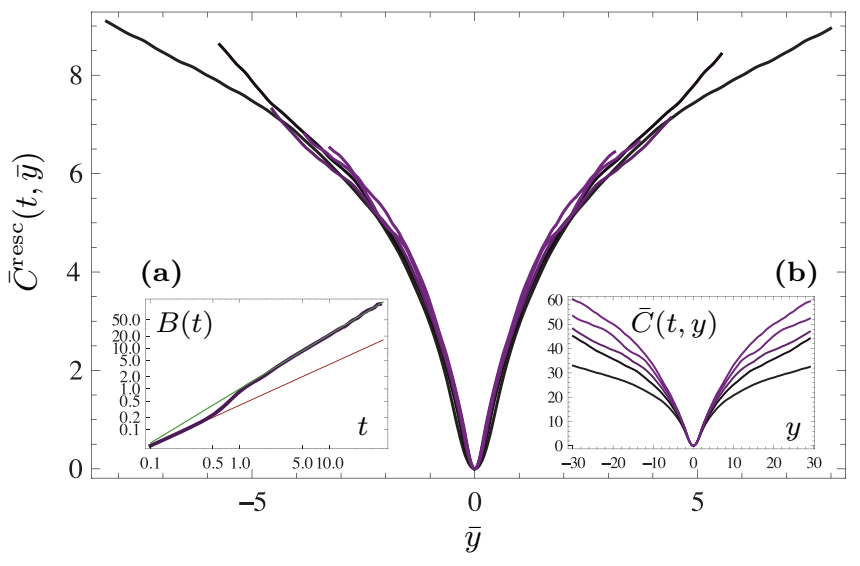

FIG. 12. (Color online) Graph for the continuous DP of the rescaled correlator $\bar{C}^{\text {resc }}(t, \bar{y})$ defined in Eq. (45) as a function of $\bar{y}=y / \sqrt{B(t)}$ for different times $t$, at low temperature. Parameters are $c=1, D=8, T=0.4$, and $\xi=2$. Disorder average is performed over 1092 realizations. (a) Roughness $B(t)$ in log-log scale with the thermal [red line (bottom)] and random manifold [green line (top)] asymptotic regimes. (b) Corresponding bare correlator $\bar{C}(t, y)$. Time varies from 35 to 135 by steps of 25 from bottom to top curves.

\section{B. Results}

For numerical simplicity, the scaling (22) was tested graphically at fixed $\xi$, defining a rescaled transverse coordinate $\bar{y}=y / \sqrt{B(t)}$ and a rescaled correlator

$$
\bar{C}^{\text {resc }}(t, \bar{y})=\frac{1}{\sqrt{B(t)}} \bar{C}_{\xi}(t, \bar{y} \sqrt{B(t)}) .
$$

Indeed, testing the full scaling (22) where $\xi$ is also rescaled by $\sqrt{B(t)}$ would imply measuring $\bar{C}_{\xi}(t, y)$ for many different values of $\xi$, which was not numerically accessible [see below for an analytical explanation on the rescaling $\xi / \sqrt{B(t)}$ of (22)]. Note that in the discrete DP it was sufficient to study the scaling function of (40) without taking $\xi$ into account (no length below the lattice spacing, which plays the role of $\xi$, can be considered).

Low-temperature results are shown in Fig. 12: The curves of $\bar{C}_{\xi}(t, y)$ at different times superimpose upon the rescaling (45), with a slight discrepancy around the origin due to the fixed disorder correlation length $\xi$. At higher temperature (Fig. 13) this scaling remains valid, the effect of the finite $\xi$ being as expected less important. In the evaluation of $\bar{C}^{\text {resc }}(t, \bar{y})$ from (45), the roughness $B(t)$ is determined from the numerical results for $\bar{F}(t, y)$ using (5) to evaluate the thermal average $\left\langle y(t)^{2}\right\rangle_{V}$ at fixed disorder, averaging afterwards over the realizations of disorder. For completeness, the graphs of the roughness are given in the inset (a) of Figs. 12 and 13 .

Similarly to the case of the discrete polymer (41), it can be argued that the $(c, D, T)$ dependence of the scaling law (22) can be absorbed into a single prefactor $\widetilde{D}[58]$ :

$$
\bar{C}_{\xi}(t, y)=\widetilde{D} \sqrt{B(t)} \hat{C}_{\frac{\xi}{\sqrt{B(t)}}}^{1}\left(\frac{y}{\sqrt{B(t)}}\right),
$$

where the function $\hat{C}_{\bar{\xi}}^{1}(\bar{y})$ does not depend on $(c, D, T)$. By tuning appropriately $\widetilde{D}$, we show on Fig. 14 from the

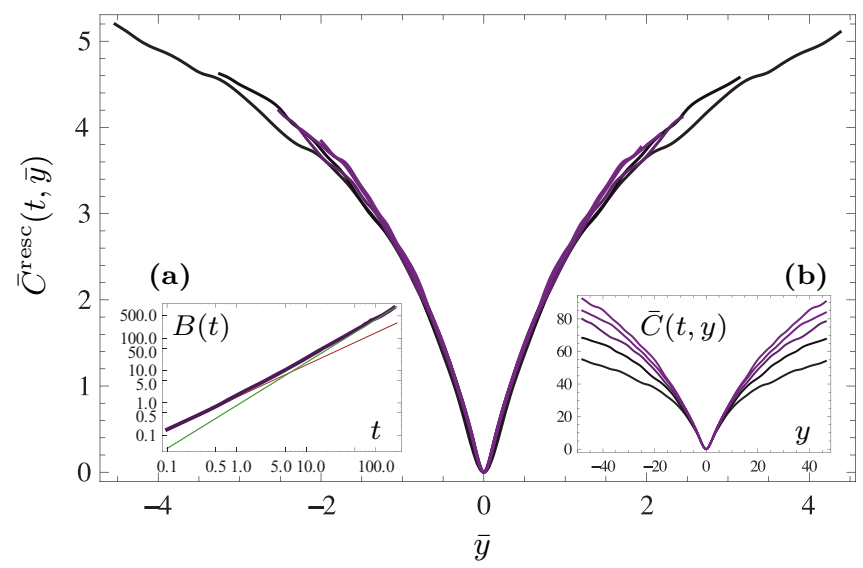

FIG. 13. (Color online) Same graph as Fig. 12 but at higher temperature $T=1.5$ (disorder average is over 446 realizations).

numerical evaluation of the correlator that this is indeed the case. The function $\widetilde{D}(T)$ at fixed $c$ and $\xi$ is evaluated by fixing a reference curve $\bar{C}^{\text {resc }}(t, \bar{y})$ at $T=T_{\text {ref }}\left(T_{\text {ref }}=2\right.$ in Fig. 14) and finding the best $\widetilde{D}(T)$ which minimizes the distance between $\bar{C}^{\text {resc }}(t, \bar{y})$ and the reference curve using the least-square method. We checked that the result does not depend on the choice of $T_{\text {ref }}$ or on the choice of the fixed $t$, within numerical uncertainty. This method allows a determination of $\widetilde{D}(T)$ up to a $T$-independent constant which imposes $\widetilde{D}\left(T_{\text {ref }}\right)=1$. We refer the reader to Ref. [58] for an in-depth analytical and numerical study of $\widetilde{D}$, regarding its scaling and physical interpretation. As displayed in the inset (b) of Fig. 14, the measured $\underset{\widetilde{D}}{ }$ is compatible with the predicted high $T$ behavior $\widetilde{D}=\frac{c D}{T}$ and a saturation at low $T$.

We can actually provide a short argument explaining the rescaling of the correlation length $\xi$ by $\sqrt{B(t)}$ appearing in Eq. (46), which we could not probe numerically. As the

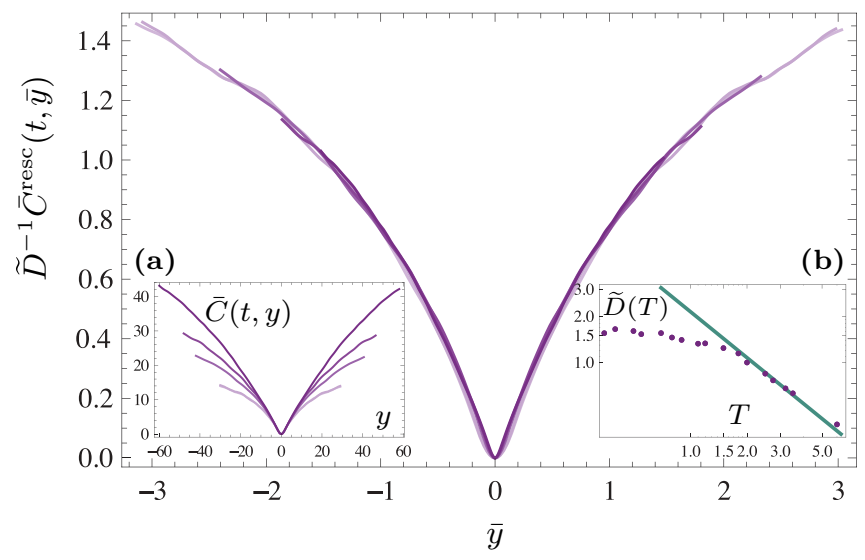

FIG. 14. (Color online) Plot of the rescaled correlator $\widetilde{D}^{-1} \bar{C}^{\text {resc }}(t, \bar{y})$ as a function of $\bar{y}$ at fixed large time $t$ and for different temperatures $T \in\{.4, .55,1.2,1.5,1.8\}$. (a) Corresponding original correlator $\bar{C}(t, y)$. Lighter color corresponds to lower temperature. (b) $\widetilde{D}$ as a function of $T$ (up to a numerical factor) in log-log scale. Points are determined by least-square minimization (see text) with respect to a reference curve at $T=2$ which fixes $\left.\widetilde{D}\right|_{T=2}=1$. The line gives the expected slope of the large temperature asymptotics $\widetilde{D} \propto \frac{1}{T}$. 
rounding of $\bar{C}(t, y)$ is quadratic in $y$ for small $y$ [see also (C21)], we expect that $\hat{C}_{\xi}^{1}(\bar{y})$ scales as

$$
\hat{C}_{\bar{\xi}}^{1}(\bar{y}) \simeq \widetilde{D} \frac{\bar{y}^{2}}{\bar{\xi}} \quad \text { for } \quad|\bar{y}| \ll(\bar{\xi} / \widetilde{D})^{1 / 2} .
$$

The rescaling $\bar{\xi}=\xi / \sqrt{B(t)}$ in Eq. (46) ensures thus that, in the large time limit of (46), $\bar{C}_{\xi}(t, y)$ remains independent of $t$ at small $y$ :

$$
\bar{C}_{\xi}(t, y) \underset{t \rightarrow \infty}{\stackrel{y \approx 0}{\longrightarrow}} \frac{\widetilde{D}}{2 \xi \sqrt{\pi}} y^{2} .
$$

Without the precise rescaling $\xi / \sqrt{B(t)}$ in Eq. (46) this expression would become singular.

Note that because of the rounding at small $y$ the fitting procedure of $\hat{C}^{1}(t, y)$ with respect to $g_{\mathrm{PS}}$, as done for the discrete DP in Fig. 11, cannot be performed since the Airy $_{2}$ process is not adapted to a finite disorder correlation length $\xi$.

\section{DISCUSSION AND CONCLUSION}

In the one-dimensional KPZ universality class, a suitably centered observable $\bar{F}(t, y)$ presents, in the large time limit, universal fluctuations of order $t^{\frac{1}{3}}$ at a transverse scale $y \sim t^{\frac{2}{3}}$, described by the scaling relation (1). We have probed numerically a refinement of this scaling law at short times, for the case of a random environment presenting correlations at a typical finite length scale $\xi$ and at finite temperature, by testing the scaling relation (46) for the two-point free-energy correlation function $\bar{C}_{\xi}(t, y)$ defined in Eq. (16). If this scaling extends to the higher order correlation functions, it will be equivalent to stating that $\bar{F}(t, y)$ scales in distribution as

$$
\bar{F}(t, y) \sim[\widetilde{D} \sqrt{B(t)}]^{\frac{1}{2}} \chi_{\frac{\xi}{\sqrt{B(t)}}}(y / \sqrt{B(t)}) .
$$

Compared to the large time asymptotics (1), this relation expresses the fact that the transverse fluctuations $\overline{\left\langle y^{2}(t)\right\rangle}=B(t)$ correspond to free-energy fluctuations of order $\sqrt{B(t)}$, described by the roughness $B(t)$ not only in the RM regime where $B(t) \propto t^{\frac{4}{3}}$ but also at smaller times. The properly rescaled function $\chi_{\bar{\xi}}(\bar{y})$ would thus be independent of the parameters $c$, $D, T$, and $\xi$, the constant $\widetilde{D}$ capturing all the parameters dependence. We have in particular recovered numerically that the value [56] $\widetilde{D}=\frac{c D}{T}$ describes correctly the high-temperature regime where the disorder correlation length $\xi$ plays no role and correlations take the form (10). Moreover, the distribution of the variable $\chi_{\bar{\xi}}(\bar{y})$ is known at $\bar{\xi}=0\left(\chi_{0}(y)=\chi(y)\right.$ being then the Airy ${ }_{2}$ process [45]) and an interesting open question is thus to characterize the analogous process at nonzero $\bar{\xi}$. One can conjecture that a suitable characterization is provided by an appropriate Macdonald process (or a generalization of it), already known [65,78] to yield the process $\chi(y)$ at $\bar{\xi}=0$ in some specific limit, especially in view of recent results [79] allowing to represent Macdonald processes in terms of Brownian motions interacting within a finite range. This could in particular help to characterize the universality of the process $\chi \bar{\xi}(\bar{y})$, i.e., to determine how many details of the correlator $R_{\xi}(y)$ of the microscopic disorder (13) are left in $\chi_{\bar{\xi}}(\bar{y})$.

A point of particular interest is the scaling of fluctuations with respect to temperature. Regarding the roughness $B(t)$ it has been shown $[23,52,75]$ that, above a characteristic temperature $T_{c}=(\xi c D)^{\frac{1}{3}}, B(t)$ can be rescaled as

$$
\begin{gathered}
B(t ; c, D, T)=\xi_{\mathrm{th}}^{2} \hat{B}\left(t / t_{*}\right), \\
\text { with } \quad \xi_{\text {th }}=\frac{T^{3}}{c D}, \quad t_{*}=\frac{T^{5}}{c D^{2}},
\end{gathered}
$$

while below $T_{c}$ such a rescaling does not hold. Nevertheless, our results on the free-energy correlator $\bar{C}(t, y)$ indicate that the extended scaling law (46) holds below and above $T_{c}$ at large enough times, the dependence in the parameters $(c, D, T, \xi)$ being then gathered in a single prefactor $\tilde{D}$. If the roughness exponent $\zeta_{\mathrm{RM}}$ is not expected to change below $T_{c}$, however, the prefactor $\hat{A}$ of the roughness in $B(t) \sim \hat{A} t^{2 \zeta_{\mathrm{RM}}}$ can be modified by $\xi$. This question is relevant in particular to determine the precise dependence of the crossover timescales of one-dimensional interfaces described by the DP coordinate $y(t)[23,53]$. We refer the reader to Ref. [58] for numerical and analytical results in that direction.

On the other hand, recent experiments on liquid crystals [42-44] (see also Ref. [9] for a burning front experiment) have demonstrated that the height fluctuations of a growth interface are correctly described by the (centered) free energy $\bar{F}(t, y)$ itself. In particular it was found that different Airy processes of the KPZ universality class successfully account for the observed height distribution (Airy ${ }_{1}$ or Airy depending $_{2}$ on the geometry of the initial condition). The authors of those studies were in particular able to measure the correlator $\bar{C}(t, y)$ with high precision. Given the existence of finite disorder correlation length $\xi$ in experiments, it would be interesting to study the equivalent of the low- and high-temperature regimes when tuning the system parameters which plays the role of $T$ in our description. In the notation of Ref. [44], the relation between the amplitude $\Gamma$ of $\chi(y)$ [linked to our $\widetilde{D}$ in Eq. (49) by $\left.\Gamma=\widetilde{D}^{2} / c\right]$ and $\lambda$ and $v$ in the KPZ equation (linked to our parameters by $c=\frac{1}{\lambda}$ and $T=\frac{\nu}{\lambda}$ ) would change from low $v$ to high $v$. Precisely, defining the characteristic value $v_{c}=\left(\xi \lambda^{2} D\right)^{\frac{1}{3}}$, we predict a crossover from the (observable) dependence $\Gamma \sim D^{2} \lambda v^{-2}$ at high $v\left(v \gg v_{c}\right)$ to a saturated regime $\Gamma \sim D^{2} \lambda v_{c}^{-2}$ at low $v\left(v \ll v_{c}\right)$. The influence of $\xi$ could thus be probed by measuring the power-law dependence of $\Gamma$ in the parameter $\lambda$ displaying a nontrivial dependence $\Gamma \sim \lambda^{-\frac{1}{3}}$ for $v \ll v_{c}$.

\section{ACKNOWLEDGMENTS}

We would like to thank Christophe Berthod for his help on the Mafalda cluster at the University of Geneva, where part of the simulations were run, and Folkmar Bornemann for sharing with us his data for the two-point correlation of the Airy ${ }_{2}$ process. E.A. and T.G. acknowledge support by the Swiss National Science Foundation under MaNEP and Division II. V.L. was financially supported by ANR SHEPI. 
G.S. acknowledges support by ANR grant 2011-BS04-013-01 WALKMAT. S.B. and G.S. acknowledge partial support by the France-Argentina MINCYT-ECOS A08E03. S.B. is partially supported by CONICET grant PIP11220090100051.

\section{APPENDIX A: DIFFERENT FORMULATIONS OF THE FREE-ENERGY CORRELATOR}

We determine in this Appendix some properties of the freeenergy correlator $\bar{C}_{\xi}(t, y)$ defined in Eq. (16). Defined by the decomposition (15) of the free energy, the reduced free energy $\bar{F}(t, y)$ is statistically invariant by translation along $y$, which implies that all its moments are invariant by translation along $y$ at fixed time:

$$
\overline{\bar{F}_{V}\left(t, y_{1}+Y\right) \ldots \bar{F}_{V}\left(t, y_{n}+Y\right)}=\overline{\bar{F}_{V}\left(t, y_{1}\right) \ldots \bar{F}_{V}\left(t, y_{n}\right)} .
$$

In particular, $\overline{\bar{F}(t, y)}$ is independent of $y$, so that $\bar{C}_{\xi}(t, y)$ is also the connected correlator of $F_{V}(t, y)$. Indeed, starting from (15)

$$
\begin{aligned}
\overline{F_{V}(t, y)} & =\overline{c \frac{y^{2}}{2 t}+\frac{T}{2} \log \frac{2 \pi T t}{c}+\bar{F}_{V}(t, y)} \\
& =c \frac{y^{2}}{2 t}+\operatorname{const}(t)
\end{aligned}
$$

thus

$$
\begin{aligned}
\bar{F}_{V}(t, y) & =F_{V}(t, y)-c \frac{y^{2}}{2 t}-\frac{T}{2} \log \frac{2 \pi T t}{c} \\
& =F_{V}(t, y)-\overline{F_{V}(t, y)}+\operatorname{const}(t),
\end{aligned}
$$

and hence from the definition (16), and dropping the index $V$ for simplicity

$$
\begin{aligned}
\bar{C}_{\xi}\left(t, y^{\prime}-y\right) & =\overline{\left\{\left[F\left(t, y^{\prime}\right)-\overline{F\left(t, y^{\prime}\right)}\right]-[F(t, y)-\overline{F(t, y)}]\right\}^{2}} \\
& =\overline{\left[F\left(t, y^{\prime}\right)-F(t, y)\right]^{2}}-\left[\overline{F\left(t, y^{\prime}\right)-F(t, y)}\right]^{2},
\end{aligned}
$$

as announced. The fact that this expression depends only on $y^{\prime}-y$ is not obvious and arises from the STS through (15). See Ref. [58] for a study of the time dependence of the quantity $\overline{\bar{F}_{V}(t, y)}$, which is independent of $y$.
Note that the second derivative of $\bar{C}_{\xi}(t, y)$ with respect to $y$ is directly related to the correlator of the "phase" $\eta_{V}(t, y) \equiv \partial_{y} \bar{F}_{V}(t, y)$. Indeed, using $\bar{F}_{V}(t, y)-\bar{F}_{V}(t, 0)=$ $\int_{0}^{y} d y_{1} \eta_{V}\left(t, y_{1}\right)$ one has

$$
\begin{aligned}
\partial_{y}^{2} \bar{C}_{\xi}(t, y) & =\partial_{y}^{2} \overline{\bar{F}(t, y)-\bar{F}(t, 0)]^{2}} \\
& =\partial_{y}^{2} \int_{0}^{y} d y_{1} \int_{0}^{y} d y_{2} \overline{\eta_{V}\left(t, y_{1}\right) \eta_{V}\left(t, y_{2}\right)} \\
& =\partial_{y}^{2} \int_{0}^{y} d y_{1} \int_{0}^{y} d y_{2} \bar{R}\left(t, y_{2}-y_{1}\right) \\
& =\bar{R}(t, y)+\bar{R}(t,-y), \\
\partial_{y}^{2} \bar{C}_{\xi}(t, y) & =2 \bar{R}(t, y),
\end{aligned}
$$

where the correlator of $\eta_{V}$ is denoted by

$$
\bar{R}\left(t, y_{2}-y_{1}\right)=\overline{\eta_{V}\left(t, y_{1}\right) \eta_{V}\left(t, y_{2}\right)} .
$$

\section{APPENDIX B: LARGE $y$ BEHAVIOR OF THE CORRELATOR $\bar{C}_{\xi}(t, y)$}

In this Appendix, we show that, as discussed in Sec. III A, the correlator $\bar{C}(t, y)$ presents "wings" at all finite times, i.e., goes to a finite constant $\lim _{|y| \rightarrow \infty} \bar{C}(t, y)$ at large $|y|$ for $t<\infty$.

To this aim, let us first show that the integral $\mathcal{N}(t)=\int d y \bar{R}(t, y)$ of the two-point correlator of $\eta_{V}(t, y)$, defined in Eq. (A13), is a conserved quantity. From (7), the evolution equation for the reduced free energy $\bar{F}_{V}(t, y)$ of the directed polymer, defined in Eq. (15), is given by (43)

$$
\begin{aligned}
\partial_{t} \bar{F}_{V}(t, y)= & \frac{1}{2 \beta c} \partial_{y}^{2} \bar{F}_{V}(t, y)-\frac{1}{2 c}\left[\partial_{y} \bar{F}_{V}(t, y)\right]^{2} \\
& -\frac{y}{t} \partial_{y} \bar{F}_{V}(t, y)+V(t, y)
\end{aligned}
$$

so that the evolution equation of $\eta_{V}(t, y)=\partial_{y} \bar{F}_{V}(t, y)$ also includes an explicit time dependence:

$$
\begin{aligned}
\partial_{t} \eta_{V}(t, y)= & \frac{1}{2 \beta c} \partial_{y}^{2} \eta_{V}(t, y)-\frac{1}{c} \eta_{V}(t, y) \partial_{y} \eta_{V}(t, y) \\
& -\frac{1}{t} \eta_{V}(t, y)-\frac{y}{t} \partial_{y} \eta_{V}(t, y)+\partial_{y} V(t, y) .
\end{aligned}
$$

One can now compute $\partial_{t} \int d y \bar{R}(t, y)$. Taking advantage of the invariance of the disorder distribution through the symmetry by reflection $y \mapsto-y$, and of the statistical invariance of $\eta_{V}(t, y)$ by translation along $y$, one has, dropping the index $V$ for simplicity,

$$
\begin{aligned}
\partial_{t} \int d y \bar{R}(t, y)= & \partial_{t} \int d y \overline{\eta(t, y) \eta(t, 0)} \\
= & \int d y \overline{\left\{\eta(t, y)\left[\frac{1}{2 \beta c} \partial_{y}^{2} \eta(t, 0)-\frac{1}{c} \eta(t, 0) \partial_{y} \eta(t, 0)-\frac{1}{t} \eta(t, 0)+\partial_{y} V(t, 0)\right]\right.} \\
& \left.+\eta \overline{(t, 0)[\frac{1}{2 \beta c} \partial_{y}^{2} \eta(t, y)-\frac{1}{c} \eta(t, y) \partial_{y} \eta(t, y) \underbrace{-\frac{1}{t} \eta(t, y)-\frac{y}{t} \partial_{y} \eta(t, y)}_{=-\frac{1}{t} \partial_{y}[y \eta(t, y)]}+\partial_{y} V(t, y)]}\right\}
\end{aligned}
$$




$$
\begin{aligned}
= & -\frac{1}{t} \int d y \overline{\eta(t, y) \eta(t, 0)}+\frac{1}{\beta c} \overline{\eta(t, 0)\left[\partial_{y} \eta(t, y)-\partial_{y} \eta(t, 0)\right]_{y=-\infty}^{y=+\infty}} \\
& -\frac{1}{c} \overline{\eta(t, 0)\left[\eta(t, y)^{2}\right]_{y=-\infty}^{y=+\infty}}-\frac{1}{t} \overline{\eta(t, 0)[y \eta(t, y)]_{y=-\infty}^{y=+\infty}} \\
\Longleftrightarrow & \partial_{t} \int d y \bar{R}(t, y)=-\frac{1}{t} \int d y \bar{R}(t, y) .
\end{aligned}
$$

This shows that $\mathcal{N}(t)$ verifies the equation $\partial_{t} \mathcal{N}(t)=-\frac{1}{t} \mathcal{N}(t)$ whose solution reads $\mathcal{N}(t)=\frac{C_{1}}{t}$. The constant $C_{1}$ is equal to 0 due to the initial condition $\mathcal{N}(0)=0$ [the initial condition for $\bar{F}$ is $\bar{F}_{V}(0, y)=0$, see Sec. III A, and thus $\eta_{V}(0, y)=0$ ]. This yields that $\mathcal{N}(t)$ is constant in time and equal to 0 :

$$
\mathcal{N}(t)=\int d y \bar{R}(t, y)=0 .
$$

This equality at all finite times $t$ is equivalent to the existence of wings in the correlator $\bar{C}_{\xi}(t, y)$ at finite times. Indeed, since $2 \int_{0}^{y} d y^{\prime} \bar{R}\left(t, y^{\prime}\right)=\partial_{y} \bar{C}_{\xi}(t, y)$ [see (A12)], this equality also writes

$$
\lim _{y \rightarrow \infty} \partial_{y} \bar{C}_{\xi}(t, y)=0,
$$

and this corresponds to the plateau at large $|y|$ of Fig. 3. This result is already known to hold at large finite time and for a delta-correlated disorder $(\xi=0)$ [45]. In this Appendix we thus have generalized this result at all times, and to a disorder correlator with short-range correlations.

\section{APPENDIX C: SHORT-TIME DYNAMICS (DIFFUSIVE SCALING)}

In this Appendix we study in the short-time regime and at finite $\xi$ the scaling behavior of the two-point correlators $\bar{C}_{\xi}(t, y)$ and $\bar{R}_{\xi}(t, y)$ of $\bar{F}_{V}(t, y)$ and $\eta_{V}(t, y)=\partial_{y} \bar{F}_{V}(t, y)$, defined respectively in Eqs. (16) and (A13).

The polymer is pinned in $y=0$ at time $t=0$ so that the initial condition translates for the reduced free energy into $\bar{F}_{V}(0, y)=0$. Let us thus assume that $\bar{F}_{V}(0, y)$ remains small at short times so that in this regime the nonlinear term of (B1) remains small and negligible compared to the linear terms:

$$
\partial_{t} \bar{F}_{V}(t, y) \simeq \frac{T}{2 c} \partial_{y}^{2} \bar{F}_{V}(t, y)-\frac{y}{t} \partial_{y} \bar{F}_{V}(t, y)+V(t, y) .
$$

This equation is linear and can thus be solved directly. Before doing so, note that for an uncorrelated disorder $(\xi=0)$, the corresponding steady-state distribution of $\bar{F}$ is the same [56] as in the nonlinearized one (9) [remark from (15) that $F$ and $\bar{F}$ share the same distribution at infinite time]. In particular, denoting $\bar{C}_{\xi}^{\operatorname{lin}}(t, y)$ [resp. $\left.\bar{R}_{\xi}^{\operatorname{lin}}(t, y)\right]$ the same correlator as (16) [resp. (A13)] but for $\bar{F}_{V}$ solution of the linearized equation (C1), one also has

$$
\begin{gathered}
\left.\bar{C}_{\xi}^{\operatorname{lin}}(t, y)\right|_{\xi=0} \underset{t \rightarrow \infty}{\longrightarrow} \frac{c D}{T}|y|, \\
\left.\bar{R}_{\xi}^{\operatorname{lin}}(t, y)\right|_{\xi=0} \underset{t \rightarrow \infty}{\longrightarrow} \frac{c D}{T} \delta(y),
\end{gathered}
$$

where $\bar{R}_{\xi}^{\operatorname{lin}}(t, y)=\frac{1}{2} \partial_{y}^{2} \bar{C}_{\xi}^{\operatorname{lin}}(t, y)$ as in Eq. (A12). Although the solution of the linearized evolution equation $(\mathrm{C} 1)$ is valid only at short time compared to the complete evolution (B1), it is thus instructive to study its behavior at all times.

In what follows, we determine the finite $t$ and finite $\xi$ equivalents of the correlators (C2) and (C3). To get rid of the term $\frac{y}{t} \partial_{y} \bar{F}_{V}$ in Eq. (C1) we set

$$
\bar{F}_{V}(t, y)=\sqrt{t} e^{\beta \frac{c y^{2}}{2 t}} \hat{F}_{V}(t, y) .
$$

The evolution of $\hat{F}_{V}$ is then

$$
\partial_{t} \hat{F}_{V}(t, y)=\frac{1}{2 \beta c} \partial_{y}^{2} \hat{F}_{V}(t, y)+\underbrace{\frac{1}{\sqrt{t}} e^{\frac{-\beta c y^{2}}{2 t}} V(t, y)}_{\equiv \hat{V}(t, y)} .
$$

Besides, in absence of disorder $(\hat{V}=0)$,

$$
G(t, y)=\sqrt{\frac{\beta c}{2 \pi t}} e^{-\frac{\beta c y^{2}}{2 t}} \theta(t)
$$

is a Green function of the equation for $\hat{F}_{V}$, that is,

$$
\left[\partial_{t}-\frac{1}{2 \beta c} \partial_{y}^{2}\right] G\left(t-t^{\prime}, y-y^{\prime}\right)=\delta\left(t-t^{\prime}\right) \delta\left(y-y^{\prime}\right) .
$$

We denote by $\theta(t)$ the Heaviside step function. The solution of (C5) is hence

$$
\hat{F}(t, y)=\int_{0}^{+\infty} d t^{\prime} \int d y^{\prime} G\left(t-t^{\prime}, y-y^{\prime}\right) \hat{V}\left(t^{\prime}, y^{\prime}\right) .
$$

Here and in what follows, integrals along direction $y$ run by convention on the real line.

Let us first determine the effect of $\xi$ by computing the value of the peak of the correlator

$$
\begin{aligned}
\bar{R}_{\xi}^{\operatorname{lin}}(t, 0) & =\left.\frac{1}{2} \partial_{y}^{2} \overline{[\bar{F}(t, y)-\bar{F}(t, y)]^{2}}\right|_{y=0} \\
& =\overline{\partial_{y} \bar{F}(t, 0) \partial_{y} \bar{F}(t, 0)}
\end{aligned}
$$

which is a matter of Gaussian integration. One has $\partial_{y} \bar{F}(t, 0)=$ $\sqrt{t} \partial_{y} \hat{F}(t, 0)$ and

$$
\begin{aligned}
\partial_{y} \hat{F}(t, 0)= & \int_{0}^{+\infty} d t_{1} \int d y_{1} \partial_{y} G\left(t-t_{1},-y_{1}\right) \hat{V}\left(t_{1}, y_{1}\right) \\
= & \frac{1}{\sqrt{2 \pi}} \int_{0}^{t} d t_{1} \int d y_{1}\left(\frac{\beta c}{t-t_{1}}\right)^{\frac{3}{2}} y_{1} e^{-\frac{\beta c y_{1}^{2}}{2\left(t-t_{1}\right)}} \\
& \times \frac{e^{\frac{-\beta c y_{1}^{2}}{2 t_{1}}}}{\sqrt{t_{1}}} V\left(t_{1}, y_{1}\right)
\end{aligned}
$$

so that putting everything together, and considering for the disorder $V$ the Gaussian correlator $R_{\xi}^{\text {Gauss }}(y)$ defined in 
Eq. (14), one gets

$$
\begin{aligned}
& \bar{R}_{\xi}^{\operatorname{lin}}(t, 0)=t \overline{\partial_{y} \hat{F}(t, 0) \partial_{y} \hat{F}(t, 0)}
\end{aligned}
$$

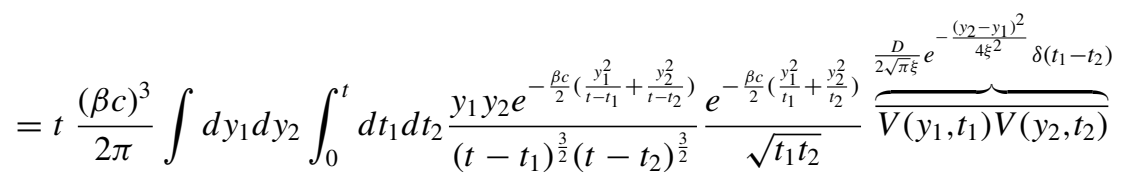

$$
\begin{aligned}
& =t \frac{(\beta c)^{3}}{4 \pi^{\frac{3}{2}} \xi} D \int d y_{1} d y_{2} \int_{0}^{t} d t_{1} \frac{y_{1} y_{2}}{\left(t-t_{1}\right)^{3} t_{1}} \exp \left[-\frac{\beta c}{2}\left(\frac{1}{t-t_{1}}+\frac{1}{t_{1}}\right)\left(y_{1}^{2}+y_{2}^{2}\right)-\frac{\left(y_{2}-y_{1}\right)^{2}}{4 \xi^{2}}\right] \text {. }
\end{aligned}
$$

The quadratic form in the last exponential writes $-\frac{1}{2} \vec{y}^{T} A \vec{y}$ with $\vec{y}=\left(y_{1}, y_{2}\right)$ and $A$ the matrix

$$
A=\left(\begin{array}{lc}
\beta c\left(\frac{1}{t_{1}}+\frac{1}{t-t_{1}}\right)+\frac{1}{2 \xi^{2}} & -\frac{1}{2 \xi^{2}} \\
-\frac{1}{2 \xi^{2}} & \beta c\left(\frac{1}{t_{1}}+\frac{1}{t-t_{1}}\right)+\frac{1}{2 \xi^{2}}
\end{array}\right) .
$$

The result of the Gaussian integral with respect to $\vec{y}$ is

$$
\left(A^{-1}\right)_{12} \sqrt{\operatorname{det} \frac{2 \pi}{A}}=\frac{\pi \xi t_{1}^{3}\left(t-t_{1}\right)^{3}}{\left\{\beta c t\left[\beta c \xi^{2} t+t_{1}\left(t-t_{1}\right)\right]\right\}^{3 / 2}},
$$

and finally (C14) becomes

$$
\begin{aligned}
\bar{R}_{\xi}^{\operatorname{lin}}(t, 0) & =t \frac{(\beta c)^{3}}{4 \pi^{\frac{3}{2}} \xi} D \int_{0}^{t} d t_{1} \frac{\pi \xi t_{1}^{2}}{\left\{\beta c t\left[\beta c \xi^{2} t+t_{1}\left(t-t_{1}\right)\right]\right\}^{3 / 2}} \\
& =\beta c D \frac{t\left(t+2 \beta c \xi^{2}\right)-\xi \sqrt{\beta c t}\left(t+4 \beta c \xi^{2}\right) \operatorname{arccot}\left(2 \xi \sqrt{\frac{\beta c}{t}}\right)}{2 \sqrt{\pi} t \xi\left(t+4 \beta c \xi^{2}\right)} .
\end{aligned}
$$

We see on one hand that the infinite time limit reads

$$
\lim _{t \rightarrow \infty} \bar{R}_{\xi}^{\operatorname{lin}}(t, 0)=\frac{c D}{2 T \xi \sqrt{\pi}},
$$

which diverges indeed as $\xi \rightarrow 0$. The effect of $\xi$ is to regularize the $\xi=0$ result $(\mathrm{C} 3)$ around $y=0$. The factor $\frac{1}{2 \sqrt{\pi}}$ directly arises from the Gaussian correlator (14) as it will become clear below. On the other hand, the short-time behavior writes

$$
\bar{R}^{\operatorname{lin}}(t, 0)=\frac{D t}{12 \xi^{2} \sqrt{\pi}}+O\left(t^{3 / 2}\right)
$$

As for the correlator $\bar{C}_{\xi}^{\operatorname{lin}}(t, y)$ of the reduced free energy, this yields from (A12) the small $y$ expansions at large and short times:

$$
\begin{array}{cc}
\bar{C}_{\xi}^{\operatorname{lin}}(t, y) \underset{t \rightarrow \infty}{\longrightarrow} \frac{c D}{2 T \xi \sqrt{\pi}} y^{2} \text { for } y \ll \sqrt{\frac{T \xi}{c D}}, \\
\bar{C}_{\xi}^{\operatorname{lin}}(t, y) \stackrel{(t \rightarrow 0)}{\approx} \frac{D t}{12 \xi^{2} \sqrt{\pi}} y^{2} & \text { for } y \ll \sqrt{\frac{\xi^{2}}{D t}} .
\end{array}
$$

The full $(t, y)$ scaling of the correlator $\bar{C}_{\xi}^{\text {lin }}(t, y)$ can be determined using the same approach as exposed above, starting from the solution $(\mathrm{C} 8)$ for $\hat{F}_{V}(t, y)$. One finds

$$
\bar{C}_{\xi}^{\operatorname{lin}}(t, y)=D \int_{0}^{t} d t_{1} \frac{1-\exp \left\{-\frac{\beta c t_{1}^{2} y^{2}}{4 t\left[\beta c \xi^{2} t+t_{1}\left(t-t_{1}\right)\right]}\right\}}{\sqrt{\pi} \sqrt{\frac{t_{1}\left(t-t_{1}\right)}{\beta c t}+\xi^{2}}} .
$$

To reveal the scaling of this expression, one performs the change of variable $t_{1}=t \tau$ in the integral

$$
\begin{aligned}
\bar{C}_{\xi}^{\operatorname{lin}}(t, y) & =\frac{c D}{T} \sqrt{\frac{t}{\beta c}} \int_{0}^{1} d \tau \frac{1-\exp \left\{\frac{-\beta c y^{2} \tau^{2} / t}{4\left[\xi^{2} \beta c / t+\tau(1-\tau)\right]}\right\}}{\sqrt{\pi} \sqrt{\tau(1-\tau)+\xi^{2} \beta c / t}} \\
& =\sqrt{\frac{t}{\beta c}} \bar{C}_{\xi \sqrt{\beta c / t}}(1, y \sqrt{\beta c / t})
\end{aligned}
$$

which takes the scaling form, with the thermal roughness $B_{\mathrm{th}}(t)=\frac{t}{\beta c}=\frac{T t}{c}$

$$
\bar{C}_{\xi}^{\operatorname{lin}}(t, y)=\frac{c D}{T} \sqrt{B_{\mathrm{th}}(t)} \hat{C}_{\frac{\xi}{\sqrt{B_{\mathrm{th}}(t)}}}\left[\frac{y}{\sqrt{B_{\mathrm{th}}(t)}}\right]
$$

where the scaling function is

$$
\begin{aligned}
\hat{C}_{\bar{\xi}}(\bar{y}) & =\left.\bar{C}_{\bar{\xi}}(t=1, \bar{y})\right|_{c=\beta=D=1} \\
& =\int_{0}^{1} d \tau \frac{1-\exp \left\{-\frac{\bar{y}^{2}}{4\left[\bar{\xi}^{2}+\tau(1-\tau)\right]}\right\}}{\sqrt{\pi} \sqrt{\tau(1-\tau)+\bar{\xi}^{2}}} .
\end{aligned}
$$

The relation (C25) describes a scaling form of the freeenergy correlation, with the roughness $B_{\mathrm{th}}(t)$ of the thermal regime. We discuss in Secs. IV B and VB an extension of its validity to the large time regime of the DP, with the full roughness $B(t)$ instead of $B_{\mathrm{th}}(t)$. 


\section{APPENDIX D: SOLUTION OF THE WINGED DP TOY MODEL IN THE GAUSSIAN VARIATIONAL METHOD APPROXIMATION}

As introduced in Refs. [71,80], what we call generically a DP "toy model" is essentially based on the assumption that the reduced free energy $\bar{F}_{V}(t, y)$ of the polymer endpoint (the "effective potential" it sees) has a Gaussian distribution, i.e., that it is fully determined by its mean value and its two-point correlator $\bar{C}^{\text {toy }}(t, y)$.

Following the scheme used in Ref. [53], we compute in this appendix the Gaussian-Variational-Method (GVM) approximation of a DP toy model with saturation "wings" appearing at $|y| \gtrsim \ell_{t}$ at time $t$. We check specifically that by consistency they should appear at $\ell_{t} \sim \sqrt{B(t)}$ at asymptotically large length scales, as asserted in Sec. III C.

\section{Replicas}

The replica approach allows to determine the statistical average of an observable $O(t, y)$ of the DP toy model as defined in Sec. III C from the formal expression

$$
\begin{aligned}
\overline{\langle O(t, y)\rangle} & =\lim _{n \rightarrow 0} \int_{\mathbb{R}^{n}} d y_{1} \ldots d y_{n} O\left(y_{1}\right) \overline{e^{-\beta \sum_{a=1}^{n} F^{\text {toy }\left(t, y_{a}\right)}}} \\
& =\lim _{n \rightarrow 0} \int_{\mathbb{R}^{n}} d y_{1} \ldots d y_{n} O\left(y_{1}\right) \exp \{-\beta \tilde{F}(t, \mathbf{y})\},
\end{aligned}
$$

where the replicated free energy $\tilde{F}(t, \mathbf{y})$ of $n$ copies $\mathbf{y}=\left(y_{1}, \ldots, y_{n}\right)$ of the polymer endpoint reads

$$
\tilde{F}(t, \mathbf{y})=\underbrace{\frac{c}{2 t} \sum_{a=1}^{n} y_{a}^{2}}_{\tilde{F}_{\mathrm{el}}(t, \mathbf{y})}+\underbrace{\frac{\beta}{4} \sum_{a, b=1}^{n} \bar{C}^{\mathrm{toy}}\left(t, y_{b}-y_{a}\right)}_{\tilde{F}_{\mathrm{dis}}(t, \mathbf{y})} .
$$

To obtain this expression, we have used the assumed Gaussianity of the $\bar{F}^{\text {toy }}$ distribution, similarly to the Gaussian distribution of the random potential.

\section{Gaussian Variational Method (GVM)}

There is no known way of determining the exact roughness of the toy model. The GVM approach $[71,80]$ for the toy model (see Ref. [53] for a detailed presentation) consists in approximating the replicated free energy (D3) by a trial quadratic free energy

$$
\tilde{F}_{0}(t)=\frac{1}{2} \sum_{a, b=1}^{n} y_{a} G_{a b}^{-1}(t) y_{b}
$$

parametrized by the $n \times n$ hierarchical matrix $G^{-1}(t)$ :

$$
G_{a b}^{-1}(t)=\frac{c}{t} \delta_{a b}-\sigma_{a b}(t)
$$

with the connected part $G_{c}^{-1}(t)=\sum_{b} G_{a b}^{-1}=\frac{c}{t}$ fixed by the case in absence of disorder. The corresponding roughness is directly read from the diagonal term of its inverse matrix, combining (D2) and (D4):

$$
B(t)=T \lim _{n \rightarrow 0} G_{a a}(t) .
$$

To find the best quadratic approximation of $\tilde{F}(t, \mathbf{y})$, the extremalization conditions read for the pairs $\{a, b\}$ :

$$
\frac{\partial \mathcal{F}_{\text {var }}}{\partial G_{a b}(t)}\left[G(t) \leftrightarrow G^{-1}(t)\right]=0,
$$

where the variational physical free energy $\mathcal{F}_{\text {var }}$ i.e., after averaging over thermal fluctuations) is defined at each time $t$ as

$$
\mathcal{F}_{\text {var }}=\mathcal{F}_{0}+\left\langle\tilde{F}-\tilde{F}_{0}\right\rangle_{0}=\mathcal{F}_{0}+\left\langle\tilde{F}_{\text {el }}-\tilde{F}_{0}\right\rangle_{0}+\left\langle\tilde{F}_{\text {dis }}\right\rangle_{0},
$$

and the trial physical free energy is

$$
\mathcal{F}_{0}=-T \log Z_{0}=-\frac{T}{2} \log \operatorname{det} G+\text { const. }
$$

In those expression $\langle\cdot\rangle_{0}$ denotes the average with respect to the normalized Boltzmann weight $e^{-\beta \tilde{F}_{0}} / Z_{0}$ and $Z_{0}$ is the corresponding partition function. Let us first compute

$$
\begin{aligned}
\left\langle\tilde{F}_{\mathrm{el}}-\tilde{F}_{0}\right\rangle_{0} & =\left\langle\frac{c}{2 t} \sum_{a=1}^{n} y_{a}^{2}-\frac{1}{2} \sum_{a, b=1}^{n} y_{a} G_{a b}^{-1}(t) y_{b}\right\rangle_{0} \\
& =\frac{1}{2} \sum_{a b}\left[\frac{c}{t} \delta_{a b}-G_{a b}^{-1}(t)\right]\left\langle y_{a} y_{b}\right\rangle_{0} \\
& =\frac{1}{2} \sum_{a b}\left[\frac{c}{t} \delta_{a b}-G_{a b}^{-1}(t)\right] T G_{a b}(t) \\
& =\frac{c T}{2 t} \sum_{a=1}^{n} G_{a a}(t)+\mathrm{const},
\end{aligned}
$$

and using the Fourier transform of the correlator $\bar{C}^{\text {toy }}(t, y)$ as defined in Eq. (25):

$$
\begin{aligned}
\left\langle\tilde{F}_{\mathrm{dis}}\right\rangle_{0} & =\frac{\beta \widetilde{D}}{4} \sum_{a, b=1}^{n} \int_{\mathbb{R}} \frac{d \lambda}{2 \pi} \frac{2}{\lambda^{2}}\left\langle 1-\cos \left[\lambda\left(y_{b}-y_{a}\right)\right]\right\rangle_{0} R_{\widetilde{\xi}}^{\text {toy }}(t, \lambda) \\
& =\frac{\beta \widetilde{D}}{2} \sum_{a b} \int_{\mathbb{R}} \frac{d \lambda}{2 \pi} \frac{1-e^{-\frac{1}{2} \lambda^{2}\left\langle\left(y_{b}-y_{a}\right)^{2}\right\rangle_{0}}}{\lambda^{2}} R_{\widetilde{\xi}}^{\text {toy }}(t, \lambda) \\
& =\frac{\beta \widetilde{D}}{2} \sum_{a b} \int_{\mathbb{R}} \frac{d \lambda}{2 \pi} \frac{1-e^{-\frac{T}{2} \lambda^{2}\left(G_{a a}+G_{b b}-2 G_{a b}\right)}}{\lambda^{2}} R_{\widetilde{\xi}}^{\text {toy }}(t, \lambda) .
\end{aligned}
$$

Gathering the results (D9), (D13), and (D16) in the definition of $\mathcal{F}_{\text {var }}(t)$ (D8), we obtain an explicit expression of $\mathcal{F}_{\text {var }}[G(t)]$, and we can apply the extremalization condition (D7) for the off-diagonal terms $a \neq b$ (setting the usual notation $\widetilde{G}=G_{a a}$ ):

$$
\begin{aligned}
0 & =-\frac{T}{2} G_{a b}^{-1}-2 \times \frac{\beta \widetilde{D}}{2} \int_{\mathbb{R}} \frac{d \lambda}{2 \pi} \frac{\lambda^{2} T}{\lambda^{2}} e^{-\lambda^{2} T\left(\widetilde{G}-G_{a b}\right)} R_{\widetilde{\xi}}^{\text {toy }}(t, \lambda) \\
& =\frac{T}{2} \sigma_{a b}-\widetilde{D} \int_{\mathbb{R}} \frac{d \lambda}{2 \pi} e^{-\lambda^{2} T\left(\widetilde{G}-G_{a b}\right)} R_{\widetilde{\xi}}^{\text {toy }}(t, \lambda)
\end{aligned}
$$

since the sum $\sum_{a b}$ in Eq. (D16) contains twice $G_{a b}$ by symmetry of the matrix, hence the " $2 \times$ " in Eq. (D17). Thus for 
a continuous parametrization $\sigma(u)(u \in[0,1]$; see Ref. [81]) of the hierarchical matrix $\sigma_{a b}$, in a full replica-symmetrybreaking (full-RSB) formulation, one gets the self-consistent equation at each time $t$ :

$$
\sigma(t, u)=2 \frac{\widetilde{D}}{T} \int_{\mathbb{R}} \frac{d \lambda}{2 \pi} e^{-\lambda^{2} T[\widetilde{G}(t)-G(t, u)]} R_{\widetilde{\xi}}^{\mathrm{toy}}(t, \lambda),
$$

where $[\widetilde{G}(t), G(t, u)]$ characterizes the matrix $G(t)$ whose matrix inverse has off-diagonal coefficients $G^{-1}(t, u)=-\sigma(t, u)$.

\section{Formal solution of the equation for $\sigma(u)$}

The algebra of hierarchical matrices in the limit $n \rightarrow 0$ has been worked out in Ref. [81], and following the conventions of Ref. [53] we recall the properties needed thereafter in the derivation of the GVM solution:

$$
\begin{gathered}
\partial_{u}[\widetilde{G}-G(u)]=-\frac{\sigma^{\prime}(u)}{\left[G_{c}^{-1}+[\sigma](u)\right]^{2}} \\
{[\sigma](u)=u \sigma(u)-\int_{0}^{u} d v \sigma(v)} \\
\widetilde{G}-G(u)=\frac{1}{u} \frac{1}{G_{c}^{-1}+[\sigma](u)}-\int_{u}^{1} \frac{d v}{v^{2}} \frac{1}{G_{c}^{-1}+[\sigma](v)} \\
\widetilde{G}=\frac{1}{G_{c}^{-1}}\left[1+\int_{0}^{1} \frac{d v}{v^{2}} \frac{[\sigma](v)}{G_{c}^{-1}+[\sigma](v)}+\frac{\sigma(0)}{G_{c}^{-1}}\right],
\end{gathered}
$$

where the connected term $G_{c}^{-1}(t)=c / t$. Denoting

$$
\mathcal{J}_{k}(t)=\int_{\mathbb{R}} \frac{d \lambda}{2 \pi} \lambda^{k} e^{-\lambda^{2} T[\widetilde{G}(t)-G(t, u)]} R_{\widetilde{\xi}}^{\text {toy }}(t, \lambda)
$$

the extremalization equation (D19) becomes simply $\sigma(u)=2 \beta \widetilde{D} \mathcal{J}_{0}$. Differentiating with $\partial_{u}$ and assuming that $\sigma^{\prime}(u) \neq 0$, we obtain

$$
1=\frac{2 \widetilde{D}}{\left[G_{c}^{-1}+[\sigma](u)\right]^{2}} \mathcal{J}_{2} .
$$

Isolating $G_{c}^{-1}+[\sigma](u)=\left(2 \widetilde{D} \mathcal{J}_{2}\right)^{1 / 2}$ and differentiating again with $\partial_{u}$ yields

$$
u=\frac{T \sqrt{2 \widetilde{D}}}{\left[G_{c}^{-1}+[\sigma](u)\right]^{2}} \frac{1}{2} \mathcal{J}_{2}^{-1 / 2} \mathcal{J}_{4} .
$$

One finally obtains that $\sigma(t, u)$ can be determined by solving the coupled self-consistent equations

$$
\sigma(u)=2 \beta \widetilde{D} \mathcal{J}_{0} \quad \text { and } \quad u=2^{-\frac{3}{2}} \widetilde{D}^{-\frac{1}{2}} T \frac{\mathcal{J}_{4}}{\mathcal{J}_{2}^{\frac{3}{2}}}
$$

and the definition (D24) which hides the $t$ dependence, e.g., by isolating $\widetilde{G}-G(u)$ as a function of $u$ from the second equation, and then inferring $\sigma(u)$ from the first. These equations are valid on segments of $u \in[0,1]$ where $\sigma(u)$ is not constant.

\section{Solution for Cauchy wings at large times}

Up to now the results are valid for a generic toy-model correlator $R_{\widetilde{\xi}}^{\text {toy }}(t, \lambda)$. In Ref. [53] we have worked out the case of a rounded correlator $R_{\widetilde{\xi}}^{\text {toy }}(t, \lambda)=e^{-\lambda^{2} \widetilde{\xi}^{2}}$. In order to add saturation "wings" to this correlator, we consider a Cauchy Ansatz (see Sec. III C and Fig. 3):

$$
R_{\widetilde{\xi}}^{\mathrm{toy}}(t, \lambda)=\frac{\lambda^{2}}{\lambda^{2}+\ell_{t}^{-2}} e^{-\lambda^{2} \widetilde{\xi}^{2}},
$$

where $\ell_{t}$ corresponds to the transverse length scale at which the wings start to develop. From the infinite-time result (18), we expect $\ell_{t}$ to diverge as $t$ goes to infinity, which allows us to study the large-scale regime by focusing on a small $\ell_{t}^{-1}$ expansion. However, bluntly expanding $R_{\widetilde{\xi}}^{\text {toy }}(t, \lambda)$ in powers of $\ell_{t}^{-1} \ll 1$ in Eq. (D19) yields divergent integrals; an alternative solution is rather to solve the coupled equations (D27) perturbatively in $\ell_{t}^{-1}$. Expanding $\mathcal{J}_{2}$ and $\mathcal{J}_{4}$ in powers of $\ell_{t}^{-1}$ one finds that the following equation in $X$ :

$$
u=2^{-\frac{1}{2}} \widetilde{D}^{\frac{1}{2}}\left[3\left(\frac{\pi}{X}\right)^{\frac{1}{4}}+7 \ell_{t}^{-2} \pi^{\frac{1}{4}} X^{\frac{3}{4}}\right]
$$

admits $X=\widetilde{\xi}^{2}+T[\widetilde{G}(t)-G(t, u)]$ as a solution to the first order in $\ell_{t}^{-1}$. The physical solution must go in the limit $\ell_{t}^{-1} \rightarrow 0$ to the solution obtained in Ref. [53] at $\ell_{t}^{-1}=0$, which involves two RSB cutoffs $u_{*}(t) \leqslant u_{c}(\widetilde{\xi})$ such that the solution $\sigma(t, u)$ is nonconstant only for $u_{*}(t) \leqslant u \leqslant u_{c}(\widetilde{\xi})$ and times below the Larkin time $t_{c}$ defined by $u_{*}\left(t_{c}\right)=u_{c}(\tilde{\xi})$. We have

$$
\begin{aligned}
\widetilde{\xi}^{2} & +T[\widetilde{G}(t)-G(t, u)] \\
& =\frac{3^{4} \pi T^{4}}{2^{8} \widetilde{D}^{2} u^{4}}+\frac{3^{7} \times 7 \pi^{2} T^{8}}{2^{10} \widetilde{D}^{4} u^{8}} \ell_{t}^{-2}+O\left(\ell_{t}^{-4}\right) .
\end{aligned}
$$

Inserting this result into the first relation in Eq. (D27), one finds that

$$
\sigma(t, u)=\frac{2}{\pi} \frac{\widetilde{D}^{2}}{T}\left(\frac{2}{3} \frac{u}{T}\right)^{2}+O\left(\ell_{t}^{-2}\right)
$$

whenever $\sigma(t, u)$ is not constant. To minimal order in $\ell_{t}^{-1}$, $\sigma(t, u)$ is translated by the constant $-\frac{\widetilde{D}}{T} \ell_{t}^{-1}$. We also see from its definition (D21) that $[\sigma](t, u)$ is not modified at this order:

$$
\begin{aligned}
{[\sigma](t, u) } & =\left.[\sigma](t, u)\right|_{\ell_{t}^{-1}=0}+O\left(\ell_{t}^{-2}\right) \\
& =\frac{2}{\pi} \widetilde{D}^{2}\left(\frac{2}{3} \frac{u}{T}\right)^{3}+O\left(\ell_{t}^{-2}\right) .
\end{aligned}
$$

Besides, the definition (D21) imposes the condition $[\sigma](t, 0)=0$ and thus the existence of $u_{*}(t)$ for which

$$
[\sigma]\left(t, u \leqslant u_{*}(t)\right)=0 .
$$

Both $\sigma(t, u)$ and $[\sigma](t, u)$ are constant in $u$ below $u_{*}(t)$ and can be strictly monotonous only above $u_{*}(t)$; this implies by continuity of $\sigma(t, u)$ that

$$
\begin{aligned}
\sigma(t, 0) & =\sigma\left(t, u \leqslant u_{*}(t)\right) \\
& =\left.\sigma(t, 0)\right|_{\ell_{t}^{-1}=0}-\frac{\widetilde{D}}{T} \ell_{t}^{-1}+O\left(\ell_{t}^{-2}\right) .
\end{aligned}
$$

To determine whether the second threshold $u_{c}(\tilde{\xi})$ has a correction which depends on $\ell_{t}^{-1}$ at this order, one has to check that the original equation (D19) is satisfied. Using the 
replica inversion formula (D22), one checks that $u_{c}$ being fixed this quantity is not modified at order $O\left(\ell_{t}^{-1}\right)$ :

$$
\widetilde{G}(t)-G(t, u)=\left.[\widetilde{G}(t)-G(t, u)]\right|_{\ell_{t}^{-1}=0}+O\left(\ell_{t}^{-2}\right),
$$

and replacing its value in the original self-consistent equation for $\sigma(t, u)$ (D19) we see that the equation on $u_{c}(\widetilde{\xi})$ is not modified at the same order.

We can hence compute the modification to the roughness $B(t)=T \lim _{n \rightarrow 0} \widetilde{G}(t)$ as given in Eq. (D6) through the formula (D23). Observing that

$$
\begin{aligned}
\widetilde{G}(t) & =\left.\widetilde{G}(t)\right|_{\ell_{t}^{-1}=0}+\frac{\sigma(t, 0)-\left.\sigma(t, 0)\right|_{\ell_{t}^{-1}=0}}{\left[G_{c}^{-1}(t)\right]^{2}}+O\left(\ell_{t}^{-2}\right) \\
& =\left.\widetilde{G}(t)\right|_{\ell_{t}^{-1}=0}-\frac{t^{2}}{c^{2}} \frac{\widetilde{D}}{T} \ell_{t}^{-1}+O\left(\ell_{t}^{-2}\right),
\end{aligned}
$$

we finally obtain

$$
B(t)=\left.B(t)\right|_{\ell_{t}^{-1}=0}-\frac{t^{2} \widetilde{D}}{c^{2}} \ell_{t}^{-1}+O\left(\ell_{t}^{-2}\right) .
$$

At asymptotically large times, we know [53] that

$$
\left.B(t)\right|_{\ell_{t}^{-1}=0}=\frac{3}{2}\left(\frac{2 \widetilde{D}^{2}}{\pi c^{4}}\right)^{\frac{1}{3}} t^{\frac{4}{3}}-\widetilde{\xi}^{2}
$$

so in the random-manifold roughness regime $B_{\mathrm{RM}}(t) \sim \frac{3}{2}\left(\frac{2 \widetilde{D}^{2}}{\pi c^{4}}\right)^{\frac{1}{3}} t^{\frac{4}{3}}$. Thus for the length scale $\ell_{t}$ not to modify the scaling of this asymptotic behavior via the correction $-\frac{t^{2} \widetilde{D}}{c^{2}} \ell_{t}^{-1}$, we must have consistently $\ell_{t} \gtrsim \sqrt{B_{\mathrm{RM}}(t)}$.

\section{APPENDIX E: FROM THE DISCRETE TO THE CONTINUOUS DP}

In this Appendix we determine formally the continuum limit of a discrete directed polymer model, whose parameters are denoted for convenience in Gothic script (the inverse temperature $\beta$, the disorder strength $\mathfrak{D}$, and when needed the elastic constant $\mathfrak{c}$ ). See also Ref. [52] for a detailed analysis of the scalings and Ref. [61] for a mathematical approach.

\section{Simplest case: Jump to the two nearest neighbors $(n=1)$}

The partition function $Z_{t, y}$ of the discrete SOS model, where the polymer can jump one step either to its right or to its left at each time step, obeys the recursion relation

$$
Z_{t, y}=e^{-\beta V_{t, y}}\left[Z_{t-1, y-1}+Z_{t-1, y+1}\right] .
$$

It is described by an inverse temperature $\beta$ and a disorder strength $\mathfrak{D}$ (hidden in $V$ through $\overline{V_{t, y} V_{t^{\prime}, y^{\prime}}}=\mathfrak{D} \delta_{t t^{\prime}} \delta_{y y^{\prime}}$ ). To explicit the correspondence between the discrete parameters $\beta$ and $\mathfrak{D}$ and the continuum parameters $\beta, c$, and $D$ [e.g., of the evolution equation (6)], we may explicit the lattice spacings $a$ and $b$ in directions $y$ and $t$, respectively:

$$
Z_{t, y}=e^{-\beta(a b \mathfrak{D})^{\frac{1}{2}} V_{t, y}^{1}}\left[Z_{t-b, y-a}+Z_{t-b, y+a}\right],
$$

where the factor $(a b \mathfrak{D})^{\frac{1}{2}}$ in front of the disorder is chosen so that $V_{t, y}^{1} \equiv(a b \mathfrak{D})^{-\frac{1}{2}} V_{t, y}$ becomes a white noise in the continuum limit:

$$
\begin{gathered}
\overline{V_{t, y}^{1} V_{t^{\prime}, y^{\prime}}^{1}}=\frac{1}{a b \mathfrak{D}} \overline{V_{t, y} V_{t^{\prime}, y^{\prime}}}=\frac{1}{b} \delta_{t, t^{\prime}} \frac{1}{a} \delta_{y, y^{\prime}} \\
\underset{a, b \rightarrow 0}{\longrightarrow} \delta\left(t-t^{\prime}\right) \delta\left(y-y^{\prime}\right) .
\end{gathered}
$$

Introducing for normalization purposes $W_{t, y}=2^{-t} Z_{t, y}$, one expands its corresponding equation of evolution as follows:

$$
\begin{aligned}
W_{t, y} & =\frac{1}{2} e^{-\beta(a b \mathfrak{D})^{\frac{1}{2}} V_{t, y}^{1}}\left[W_{t-b, y-a}+W_{t-b, y+a}\right] \\
& \simeq \frac{1}{2}\left[1-\beta(a b \mathfrak{D})^{\frac{1}{2}} V_{t, y}^{1}\right]\left[2 W_{t-b, y}+a^{2} \partial_{y}^{2} W_{t, y}\right]
\end{aligned}
$$

so that, with $W_{t, y}-W_{t-b, y} \simeq b \partial_{t} W_{t, y}$,

$$
\partial_{t} W_{t, y}=\frac{1}{2} \frac{a^{2}}{b} \partial_{y}^{2} W_{t, y}-\beta\left(a b^{-1} \mathfrak{D}\right)^{\frac{1}{2}} V_{t, y}^{1} W_{t, y},
$$

which corresponds for instance to the continuum FeynmanKac evolution (6) with the parameters

$$
\beta=\beta, \quad c=\frac{b}{a^{2}} \frac{1}{\beta}, \quad D=a b^{-1} \mathfrak{D} .
$$

This correspondence between discrete and continuum parameters is used in Sec. IV when discussing numerical results on the discrete DP.

\section{Generic case: Jump to the $2 n$ nearest neighbors}

For the sake of completeness, we now consider a generalized SOS model in the spirit of Ref. [52], where the polymer endpoint $y$ can jump to either of its $2 n$ neighbors $y-2 n+1, y-2 n+3, \ldots, y+2 n-1$ with an elastic weight depending on the distance $j$ as $e^{-\beta j^{2}}$. The partition function now obeys

$Z_{t, y}=e^{-\beta V_{t, y}} \sum_{j=1}^{n} e^{-\beta j^{2}}\left[Z_{t-1, y-(2 j-1)}+Z_{t-1, y+(2 j-1)}\right]$.

We explicit as previously the lattice spacings $a$ and $b$, and we also introduce a microscopic elastic constant $\mathfrak{c}$ :

$$
\begin{aligned}
Z_{t, y}= & e^{-\beta(a b \mathfrak{D})^{\frac{1}{2}} V_{t, y}^{1}} \sum_{j=1}^{n} e^{-\frac{1}{2} \mathfrak{c} a^{2} \beta j^{2}} \\
& \times\left[Z_{t-b, y-(2 j-1) a}+Z_{t-b, y+(2 j-1) a}\right] .
\end{aligned}
$$

Introducing, for normalization purposes, $W_{t, y}=(2 \Omega)^{-t} Z_{t, y}$ one expands its corresponding equation of evolution as follows:

$$
\begin{aligned}
W_{t, y} \simeq & \frac{1}{2 \Omega}\left[1-\beta(a b \mathfrak{D})^{\frac{1}{2}} V_{t, y}^{1}\right] \sum_{j=1}^{n} e^{-\frac{1}{2} \mathfrak{c} a^{2} \beta j^{2}} \\
& \times\left[2 W_{t-b, y}+(2 j-1)^{2} a^{2} \partial_{y}^{2} W_{t-b, y}\right] .
\end{aligned}
$$

To ensure that the dominant order of the right-hand side is $W_{t-b, y}$, which allows us to recognize a time difference, one sets

$$
\Omega=\sum_{j=1}^{n} e^{-\frac{1}{2} \mathfrak{c} a^{2} \beta j^{2}}
$$


Then, defining the effective elastic constant $\kappa_{n}(\mathfrak{c}, \beta)$ as in Ref. [52]:

$$
\kappa_{n}=\frac{\sum_{j=1}^{n} e^{-\frac{1}{2} c a^{2} \beta j^{2}}}{\beta \sum_{j=1}^{n}(2 j-1)^{2} e^{-\frac{1}{2} c a^{2} \beta j^{2}}},
$$

one recovers from (E11) the equation of evolution

$$
\partial_{t} W_{t, y}=\frac{1}{2 \beta \kappa_{n}} \frac{a^{2}}{b} \partial_{y}^{2} W_{t, y}-\beta\left(a b^{-1} \mathfrak{D}\right)^{\frac{1}{2}} W_{t, y} V_{t, y},
$$

which corresponds to the continuum evolution (6) upon the identification

$$
\beta=\beta, \quad c=\frac{b}{a^{2}} \kappa_{n}(\mathfrak{c}, \beta), \quad D=a b^{-1} \mathfrak{D} .
$$

Note that the result holds for any $n$; in particular, $\kappa_{n}=\frac{1}{\beta}$ for $n=1$ (as obtained in the previous paragraph) but $\kappa_{n}$ goes to another $\mathfrak{c}$-dependent limit for $n^{2} \gg T$, which enables us in particular to study the high-temperature limit of the discrete directed polymer model [52].
[1] S. Moulinet, C. Guthmann, and E. Rolley, Eur. Phys. J. E 8, 437 (2002).

[2] M. Alava, M. Dubé, and M. Rost, Adv. Phys. 53, 83 (2004).

[3] D. Bonn, J. Eggers, J. Indekeu, J. Meunier, and E. Rolley, Rev. Mod. Phys. 81, 739 (2009).

[4] P. Le Doussal, K. J. Wiese, S. Moulinet, and E. Rolley, Europhys. Lett. 87, 56001 (2009).

[5] M. Alava and K. Niskanen, Rep. Progr. Phys. 69, 669 (2006).

[6] P. Le Doussal, A. A. Middleton, and K. J. Wiese, Phys. Rev. E 79, 050101 (2009).

[7] P. Le Doussal and K. J. Wiese, Phys. Rev. E 79, 051106 (2009).

[8] J. Maunuksela, M. Myllys, O.P. Kähkönen, J. Timonen, N. Provatas, M. J. Alava, and T. Ala-Nissila, Phys. Rev. Lett. 79, 1515 (1997).

[9] L. Miettinen, M. Myllys, J. Merikoski, and J. Timonen, Eur. Phys. J. B 46, 55 (2005).

[10] S. Lemerle, J. Ferré, C. Chappert, V. Mathet, T. Giamarchi, and P. Le Doussal, Phys. Rev. Lett. 80, 849 (1998).

[11] V. Repain, M. Bauer, J. Jamet, J. Ferré, A. Mougin, C. Chappert, and H. Bernas, Europhys. Lett. 68, 460 (2004).

[12] P. J. Metaxas, J. P. Jamet, A. Mougin, M. Cormier, J. Ferré, V. Baltz, B. Rodmacq, B. Dieny, and R. L. Stamps, Phys. Rev. Lett. 99, 217208 (2007).

[13] T. Tybell, P. Paruch, T. Giamarchi, and J. M. Triscone, Phys. Rev. Lett. 89, 097601 (2002).

[14] P. Paruch, T. Giamarchi, and J. M. Triscone, Phys. Rev. Lett. 94, 197601 (2005).

[15] A. Barabási and H. E. Stanley, Fractal Concepts in Surface Growth (Cambridge University Press, Cambridge, 1995).

[16] J. Krug, Adv. Phys. 46, 139 (1997).

[17] G. Blatter, M. V. Feigel'man, V. B. Geshkenbein, A. I. Larkin, and V. M. Vinokur, Rev. Mod. Phys. 66, 1125 (1994).

[18] T. Halpin-Healy and Y. Zhang, Phys. Rep. 254, 215 (1995).

[19] S. Brazovskii and T. Nattermann, Adv. Phys. 53, 177 (2004).

[20] P. Le Doussal and K. J. Wiese, Phys. Rev. E 72, 035101 (2005).

[21] K. J. Wiese and P. Le Doussal, Markov Proc. Relat. Fields 13, 777 (2007).

[22] T. Giamarchi, A. Kolton, and A. Rosso, in Lecture Notes in Physics, Vol. 688 (Springer, Berlin, 2006), p. 91.

[23] E. Agoritsas, V. Lecomte, and T. Giamarchi, Physica B 407, 1725 (2012).

[24] M. Kardar, G. Parisi, and Y. C. Zhang, Phys. Rev. Lett. 56, 889 (1986).

[25] T. Sasamoto and H. Spohn, J. Stat. Mech.: Theor. Exp. (2010) P11013.
[26] I. Corwin, Random Matrices: Theory Appl. 1, 1130001 (2012).

[27] T. Kriecherbauer and J. Krug, J. Phys. A 43, 403001 (2010).

[28] D. Forster, D. R. Nelson, and M. J. Stephen, Phys. Rev. A 16, 732 (1977).

[29] F. Comets, T. Shiga, and N. Yoshida, in Stochastic Analysis on Large Scale Interacting Systems (Mathematical Society of Japan, Tokyo, 2004), pp. 115-142, http://hal.archives-ouvertes.fr/hal-00104909/.

[30] G. Giacomin, Random Polymer Models (Imperial College Press, London, 2007).

[31] F. Comets and M. Cranston, arXiv:1107.2011 (2011).

[32] K. Johansson, Comm. Math. Phys. 209, 437 (2000).

[33] M. Prähofer and H. Spohn, Phys. Rev. Lett. 84, 4882 (2000).

[34] H. Spohn, Physica A 369, 71 (2006).

[35] H. Spohn, Physica A (Amsterdam) 369, 71 (2006).

[36] J. Rambeau and G. Schehr, Europhys. Lett. 91, 60006 (2010).

[37] G. Schehr, arXiv:1203.1658 (2012).

[38] P. J. Forrester, S. N. Majumdar, and G. Schehr, Nucl. Phys. B 844, 500 (2011).

[39] M. Kulkarni and A. Lamacraft, arXiv:1201.6363 (2012).

[40] L.H. Gwa and H. Spohn, Phys. Rev. A 46, 844 (1992).

[41] H. van Beijeren, Phys. Rev. Lett. 108, 180601 (2012).

[42] K. A. Takeuchi and M. Sano, Phys. Rev. Lett. 104, 230601 (2010).

[43] K. A. Takeuchi, M. Sano, T. Sasamoto, and H. Spohn, Sci. Rep. 1, 34 (2011).

[44] K. Takeuchi and M. Sano, J. Stat. Phys. 147, 853 (2012).

[45] M. Prähofer and H. Spohn, J. Stat. Phys. 108, 1071 (2002).

[46] K. Johansson, Comm. Math. Phys. 242, 277 (2003).

[47] C. Tracy and H. Widom, Comm. Math. Phys. 159, 151 (1994).

[48] P. Calabrese, P. Le Doussal, and A. Rosso, Europhys. Lett. 90, 20002 (2010).

[49] G. Amir, I. Corwin, and J. Quastel, Comm. Pure App. Math. 64, 466 (2011).

[50] T. Sasamoto and H. Spohn, Nucl. Phys. B 834, 523 (2010).

[51] V. Dotsenko, Europhys. Lett. 90, 20003 (2010).

[52] S. Bustingorry, P. Le Doussal, and A. Rosso, Phys. Rev. B 82, 140201 (2010).

[53] E. Agoritsas, V. Lecomte, and T. Giamarchi, Phys. Rev. B 82, 184207 (2010).

[54] R. P. Feynman, Rev. Mod. Phys. 20, 367 (1948).

[55] M. Kac, Trans. Amer. Math. Soc. 65, 1 (1949).

[56] D. A. Huse, C. L. Henley, and D. S. Fisher, Phys. Rev. Lett. 55, 2924 (1985).

[57] M. Kardar, Statistical Physics of Fields (Cambridge University Press, Cambridge, 2007). 
[58] E. Agoritsas, V. Lecomte, and T. Giamarchi, arXiv:1209.0567 (2012).

[59] L. Bertini and N. Cancrini, J. Stat. Phys. 78, 1377 (1995).

[60] L. Bertini and G. Giacomin, Comm. Math. Phys. 183, 571 (1997).

[61] T. Alberts, K. Khanin, and J. Quastel, arXiv:1202.4403 (2012).

[62] M. Kardar, Nucl. Phys. B 290, 582 (1987).

[63] M. Kardar, Phys. Rev. Lett. 55, 2923 (1985).

[64] V. Dotsenko and B. Klumov, J. Stat. Mech.: Theor. Exp. (2010) P03022.

[65] A. Borodin, I. Corwin, and P. Ferrari, arXiv:1204.1024 (2012).

[66] I. Corwin, arXiv:1106.1596 (2011).

[67] T. Nattermann, Europhys. Lett. 4, 1241 (1987).

[68] G. Parisi, J. Phys. 51, 1595 (1990).

[69] M. Mézard, J. Phys. France 51, 1831 (1990).
[70] J. P. Bouchaud and H. Orland, J. Stat. Phys. 61, 877 (1990).

[71] M. Mézard and G. Parisi, J. Physique I 2, 2231 (1992).

[72] D. S. Fisher and D. A. Huse, Phys. Rev. B 43, 10728 (1991).

[73] T. Hwa and D. S. Fisher, Phys. Rev. B 49, 3136 (1994).

[74] P. Le Doussal and C. Monthus, Physica A 317, 140 (2003).

[75] T. Nattermann and W. Renz, Phys. Rev. B 38, 5184 (1988).

[76] F. Bornemann, P. L. Ferrari, and M. Prähofer, J. Stat. Phys. 133, 405 (2008).

[77] F. Bornemann, Math. Comp. 79, 871 (2010).

[78] A. Borodin and I. Corwin, arXiv:1111.4408 (2011).

[79] N. O’Connell, Ann. Probab. 40, 437 (2012).

[80] Y. Y. Goldschmidt and T. Blum, Phys. Rev. E 48, 161 (1993).

[81] M. Mézard and G. Parisi, J. Phys. I 1, 809 (1991). 